\title{
Spiral wave dynamics: reaction and diffusion versus kinematics
}

\author{
B. Fiedler, M. Georgi, and N. Jangle \\ Institut für I, Freie Universität Berlin, Arnimallee 2-6, 14195 Berlin \\ fiedler@math.fu-berlin.de, georgi@math.fu-berlin.de, \\ jangle@math.fu-berlin.de
}

\section{Phenomena}

In nature, spatio-temporal patterns in excitable media occur in seemingly unlimited variety. As early as 1946 Wiener and Rosenblueth [WR46] introduced the concept of excitable media to explain the propagation of electrical excitation fronts in the heart. Waves of electrical activity in the heart muscle assist its rhythmic contractions. The presence of spiral waves can indicate dangerous fibrillation. This is one of the motivations why the dynamics and control of spiral waves are studied. Furthermore spiral waves are typical, almost ubiquitous, patterns in excitable media; see [ZE06] in this volume. Mathematically, self-organized spiral patterns are a striking phenomenon of reaction-diffusion systems, in its own right, motivated by a large variety of application areas.

Slime mold aggregation is another example. As long as food in form of bacteria is present the slime mold cells live independently in the soil. As food becomes rare they form a multicellular "organism". This "organism" moves in order to find appropriate conditions for production and dispersal of spores. During the early phase of aggregation, chemotactic movement can proceed in form of spiral waves [FL98].

Spiral waves also arise in the oxidation of carbon-monoxide on platinum surfaces [BM03]. In 1972 they have been dicovered by Winfree [Win72] in the photosensitive Belousov-Zhabotinsky (BZ) reaction, see for recent investigations for example $\left[\mathrm{ZBB}^{+} 03, \mathrm{ZBB}^{+} 04, \mathrm{ZE04}\right]$. Both reactions are studied in the SFB 555. The classical BZ reaction is a catalytic oxidation of malonic acid, using bromate in an acidic environment. Experimentally it exhibits well reproducible drift, meander and "chaotic" motions of the spiral wave and its tip.

In several experiments and numerical simulations, transitions from rigidly rotating spiral waves to other more complicated waves have been observed. The dynamics near rigidly rotating waves and their transition to meandering and drifting spirals has been studied extensively; see, for instance 

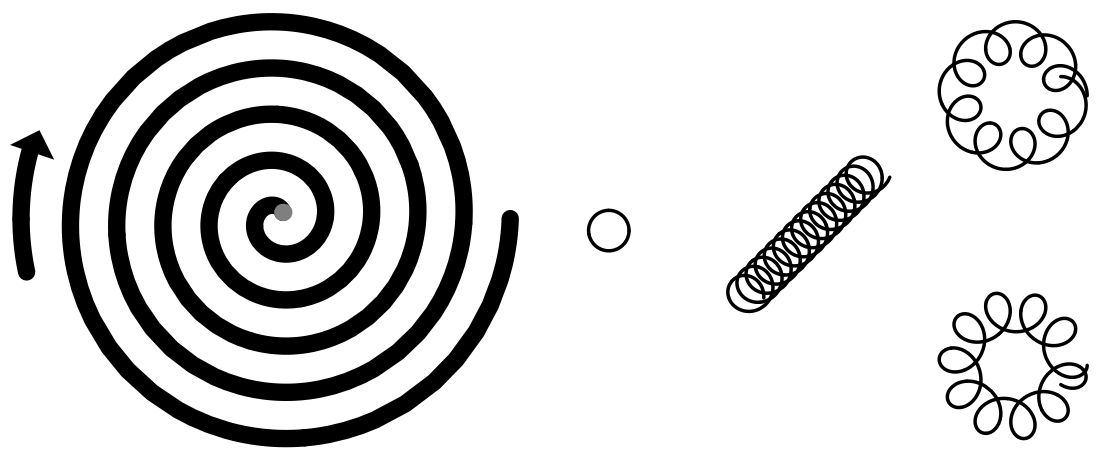

Fig. 1. Outward moving spiral wave (left) with different associated tip motions (right: rigid rotation, drift and meander).

[SS91, Bar94b, SS01]. The transition from planar meandering spirals to seemingly three frequencies has been studied numerically in [PM95] by calculating the Fourier spectrum of the waves. Analyzing the parameter space of the underlying partial differential equation, different frequency branches have been identified. These frequencies determine the coarse structure and geometry of the pattern. The effect of periodic forcing on rigidly rotating spiral wave solutions has been investigated experimentally in [NvORE93] for catalysis on platinum surfaces. The periodic forcing has been realized via an externally modulated temperature. Periodic forcing of a photosensitive variant of the BZ reaction has been studied experimentally in [BE93b, ZSM94]. The forcing is achieved by periodically changing light intensity. In both experimental settings, meanders and resonant drifts occur.

Varying the light intensity enables effective control of the motion of spiral waves, see [BBSE00, KGZM01, SZM93, ZE06]. Controlling the motion of spiral waves is an important challenge, for example, for the defibrillation of cardiac tissue. The elimination of spiral waves by multiple shocks of external current has been investigated numerically in [PZMK00].

Although the examples presented above are of very diverse nature they generate astonishingly similar phenomena. We have chosen the photosensitive $\mathrm{BZ}$ reaction as the main experimentally accessible paradigm in the SFB 555; see [ZE06] in this volume. Therefore our paper aims at the mathematical analysis of the dynamics of complex spatio-temporal patterns in nonlinear excitable, spatially or temporally heterogeneous media, with the BZ reaction in mind.

Depending on the space dimension different but related resulting dynamics can be observed. One example are the emission centers of pulse chains in one space dimension. In two space dimensions there are emissions of target patterns and the phenomena of rotating, meandering and drifting spirals. In 
three space dimensions, formally, these correspond to oscillating and drifting, possibly twisted, filaments of scroll waves.

There are two alternative methods commonly used to model the dynamics of spiral waves. The first one is a description by parabolic partial differential equations (PDEs), specifically reaction-diffusion systems. This has been one of the settings used by Wiener, originally, in [WR46]. The other method is a description of the wave fronts by reduced (mean-)curvature flows of curves and surfaces. This approach, known as kinematic theory, also dates back as far as Wiener's original paper [WR46]. The special case of an eikonal theory assumes the normal propagation velocity of the wave front to be independent of curvature, as stated in Huygens' principle and in geometric optics. For more detailed mathematical investigations, and partial justifications in a singular perturbation setting, see also [KT92], [Kee92], [MZ91], [MDZ94], the survey [Mik03], and the references there.

Either approach has advantages and disadvantages when tackling rotating, meandering and drifting spiral wave phenomena.

Reaction-diffusion systems encounter difficulties even for the seemingly simple question of mere existence of rigidly rotating spiral waves. With the technically demanding tool of spatial dynamics in the (logarithmic) radial direction, this difficulty has been overcome for small amplitude waves in a celebrated paper by Scheel [Sch98]. Subsequently, interesting consequences have been derived; most notably a first classification of possible instabilities of defect dynamics, relating to core and far-field break-up [SS04].

Moreover, under the assumption of pure point critical spectrum, a center manifold description of the associated meandering, drifting, and resonance phenomena has been achieved; see [FSSW96], [SSW99], and the survey [FS03]. We give a brief exposition in section 2.1. Specific calculations, however, require disproportionate computational effort to account for the two-dimensional time dependent problem in large (theoretically unbounded) domains.

The unbounded domain causes difficulties, on the one hand, via continuous spectrum which may - and does - interfere with the pure point spectrum required for our bifurcation analysis. The unbounded domain is necessary, on the other hand, to correctly incorporate the Euclidean symmetry of the problem under translations and rotations. In section 2 below we will specifically address competing and coexisting pinning and drifting phenomena. Such phenomena are predicted for the light-sensitive BZ system, when full Euclidean symmetry is broken towards a mere translational lattice symmetry, by choosing a spatially periodic lighting.

Kinematic theory, the alternative approach sketched in section 3, has the advantage of working with partial differential equations (PDEs) in a single space dimension: a half line which extends from the tip, at $s=0$, to the Archimedean far-field $s \rightarrow+\infty$. Here arc length $s$ parametrizes the wave front. See (40) for a PDE for curvature $\kappa=\kappa(t, s)$ as a function of time $t$ and arc length $s$. A description by curvature $\kappa(t, s)$, rather than position $(x(t, s), y(t, s))$ of the wave front, has the advantage of incorporating Eu- 
clidean equivariance into the description, a priori. Indeed, translated and rotated wave fronts are described by identical curvature functions $\kappa(t, s)$. It is therefore surprising, perhaps, that a consistent purely kinematic theory which includes meandering and drifting phenomena has remained elusive, so far. In section 3 we first derive existence and multiplicity results for rigidly rotating Archimedean spirals, fairly directly, from a center manifold argument in the associated singular second order ordinary differential equation (ODE) of the kinematic theory; see [FGT04, FGT06]. For periodic forcing of the wave speed, we then overcome the meandering/drifting barrier of the curvatureindependent eikonal theory by modification of the tip dynamics, only, following the presentation in [MDZ94]. Following [Jan06] we also derive supersipral patterns, together with a strong stability property, in the periodically forced eikonal case. In addition, we present super-superspirals of higher order, caused by nearly resonant quasi-periodic forcing functions. These eikonal results are based on constant velocity wave propagation, alone, independently of the curvature of the wave front. Such an approach has been suggested in [WR46], in the guise of wave fronts and Huygens' principle, even though the meandering/drifting effects which we now address were unknown, then.

\section{Reaction-diffusion spirals}

\subsection{Center manifold reductions}

An important feature of dynamics and bifurcations of spiral waves is the Euclidean symmetry $S E(2)$ of the plane. The special Euclidean symmetry group $S E(2)$ consists of all planar translations and rotations. Barkley [Bar94a] was the first to notice the relevance of this group for meandering spiral wave dynamics. Indeed, let $u(t, x), x \in \mathbb{R}^{2}$, be any solution of a spatially homogeneous reaction-diffusion system. Let $R$ denote any fixed rotation matrix, and $S \in \mathbb{R}^{2}$ any fixed translation vector. Then $u\left(t, R^{-1}(x-S)\right)$ is a solution of the same reaction-diffusion system. Barkley therefore proposed, but could not justify, that the dynamics of planar rigidly rotating or meandering spirals is governed by an $S E(2)$-equivariant vector field. Phenomenologically, he could then interpret the transition to meandering or drifting spiral waves as a Hopf bifurcation. Indeed, he numerically verified the crossing of a pair of simple eigenvalues of the linearization of rotating waves through the imaginary axis. Similarly, Mantel and Barkley [MB96] described periodic forcing of meandering spirals by periodically forced equivariant equations on the group $S E(2)$ itself. The mathematical tool to justify such reduced descriptions by low-dimensional systems of ODEs, which was still missing at the time, is an $S E(2)$-equivariant version of center manifold theory.

Center manifold theory has become an indispensable tool for the study of ODEs. An equilibrium is called hyperbolic if the linearization of the vector field at that equilibrium does not possess spectrum on the imaginary axis. 
The local dynamics of ODEs near a hyperbolic equilibrium is determined by that linearization. In particular there exist stable and unstable manifolds. Their tangent spaces at the equilibrium are given by the stable and unstable eigenspaces of the linearization, which are the generalized eigenspaces to eigenvalues with (strictly) negative and positive real parts, respectively. These invariant manifolds contain all solutions which locally approach the equilibrium exponentially fast in forward time and backward time, respectively. The dynamics becomes much more interesting if the equilibrium is non-hyperbolic, i.e., if the linearization possesses purely imaginary eigenvalues. Generically, conserved quantities, time reversibility, or parameters in the system may lead to purely imaginary eigenvalues. These eigenvalues give rise to a local center manifold. The tangent space of the center manifold at the equilibrium is given by the center eigenspace, which is the generalized eigenspace to the purely imaginary eigenvalues. Solutions on the center manifold have only subexponential growth or decay. Furthermore all solutions which stay bounded and small for all time are contained in the center manifold [Van89]. Local center manifolds are not unique, in contrast to stable and unstable manifolds. But center manifold theory simplifies the system. It reduces the dimension and preserves the interesting solutions. One application of center manifold theory is the Hopf bifurcation theorem. Under generic assumptions the Hopf bifurcation theorem states that an equilibrium with a simple pair of purely imaginary eigenvalues, at a specific parameter value, possesses periodic solutions on a center manifold, for nearby parameter values.

Center manifold theory extends to many infinite-dimensional systems, like certain partial differential equations (PDEs). Center manifold reductions can be obtained locally or globally. For local center manifolds of parabolic PDEs see Vanderbauwhede and Iooss [VI92]. Dimension reductions via global center manifolds for spatially inhomogeneous planar media have been achieved by Jangle [Jan03, GJ05]; more details will be presented below.

The central question, which we will pursue in the present section, asks for the consequences of symmetry breaking from full $S E(2)$-equivariance to a mere translational lattice symmetry. Experimentally this question is motivated by the photosensitive $\mathrm{BZ}$ reaction; see $\left[\mathrm{ZBB}^{+} 03, \mathrm{ZBB}^{+} 04, \mathrm{ZE} 04\right.$, ZBBE05, KM05]. In these experiments, the excitability of the BZ medium in a gel reactor depends sensitively on the intensity of light exposure. This intensity can be varied, both spatially and temporally. For time independent light intensity, for example, with slight spatial variation in form of a checker board light-dark pattern, the spatial homogeneity of the underlying reactiondiffusion system is broken to a mere lattice periodicity, spatially. Mathematically, $S E(2)$-equivariance is thus perturbed to a mere lattice symmetry. This motivates our mathematical study of the possible consequences of equivariance breaking perturbations for the dynamics of rotating wave patterns.

But what are possible dynamic consequences of such an equivariance breaking perturbation to a lattice group? As many authors before us, we describe such consequences in terms of the tip motion of meandering spi- 
rals. We would like to keep in mind, however, that the prominently visible phenomenon of the tip motion only amounts to a visualization of the translational component of the $S E(2)$ coordinates, which we will derive in section 2.2 via an associated center manifold reduction. The additional investment towards a clean, mathematically well-founded framework is amply rewarded. All ambiguities as to various ad-hoc definitions of the spiral tip, for example, are going to disappear in our setting - along with the mystery of their phenomenological equivalence.

Drifting versus pinning are the most prominent spiral tip motions in geometries with lattice symmetry. By pinning we denote tip motions which come to rest, converging to a fixed spatial location, as time $t \rightarrow+\infty$. Drifting, in contrast, indicates motions which are unbounded in the underlying lattice geometry.

In practice, of course, drifting motions will simply sweep the spiral tip to the domain boundary where the spiral will annihilate. Such boundary annihilations - a highly desirable effect in the context of defibrillation - can be understood as a cancellation with the anti-rotating mirror image spiral after local reflection through the Neumann boundary condition. Below, we do not pursue such modifications due to bounded domains.

Instead, we show below how pinning and drifting motions may coexist, within the same center manifold of the same underlying system and at the same parameter values. In fact an ever so slight variation of initial conditions may kick the solution from drifting to pinning mode, or back. The respective initial conditions for these behaviors will be interwoven in a Cantor-like structure.

More generally, we will address the question of realizing any flow on the 2-torus $T^{2}=\mathbb{R}^{2} / \mathbb{Z}^{2}$, which can and should be viewed as the space of tip positions in the plane $\mathbb{R}^{2}$ modulo the lattice periodicity $\mathbb{Z}^{2}$. The coexistence of drifting and pinning motions then becomes a corollary to the realization of Cherry flows on 2-tori, see [PdM82], by spiral tip dynamics in lattice symmetries.

\subsection{Center manifolds in unbounded domains}

Chemical systems are traditionally modeled by reaction-diffusion systems on suitable domains. As was explained above, our main modeling assumption is that the domain is actually unbounded, that is, we consider governing partial differential equations on the entire plane. This assumption may seem unrealistic: neither experiments nor numerical simulations can be performed on unbounded domains. In our particular context of spiral waves in the BZ reaction, however, experiments indicate that spiral waves behave much as if there were no boundaries. Therefore until boundary annihilation sets in - typically within only one to two wavelengths from the boundary itself - we consider reaction-diffusion systems: 


$$
\partial_{t} u(t, x)=D \Delta_{x} u(t, x)+F(u(t, x)), \quad u:[0, \infty) \times \mathbb{R}^{2} \longrightarrow \mathbb{R}^{N},
$$

where $x \in \mathbb{R}^{2}$ denotes space, $t \geq 0$ is time, and $u=u(t, x) \in \mathbb{R}^{N}$ is the concentration vector. The nonlinearity $F: \mathbb{R}^{N} \longrightarrow \mathbb{R}^{N}$ is a $C^{k+2}$-function, $k \geq 1$, and $D$ is a constant diagonal matrix with strictly positive entries.

Under appropriate growth conditions on $F$ there exists an $\alpha \in[0,1)$ such that $F \in C^{k+2}\left(X^{\alpha}, X\right)$, where $X:=L^{2}\left(\mathbb{R}^{2}, \mathbb{R}^{N}\right)$. The fractional power space $X^{\alpha}$ is an interpolation space which is more regular than $L^{2}\left(\mathbb{R}^{2}, \mathbb{R}^{N}\right)$ but less regular than the Sobolev space $H^{2}\left(\mathbb{R}^{2}, \mathbb{R}^{N}\right)$ of functions $u(t, \cdot)$ with square integrable second spatial derivatives. By Henry [Hen81], equation (1) generates a $C^{k+2}$ semiflow $\phi_{t}$ on $X^{\alpha}$. Thus the solution to equation (1) at time $t \geq 0$ with initial condition $u_{0}$ is given by a function $u=u(t, x)$ such that $u(t, \cdot)=\phi_{t}\left(u_{0}\right)$.

Equation (1) exhibits the symmetry of the homogeneous BZ reaction, namely equivariance under the Euclidean group $S E(2)$ of all translations $S$ and rotations $R$ in the plane. The group multiplication of $\left(R_{i}, S_{i}\right) \in S E(2)$ is given by

$$
\left(R_{1}, S_{1}\right)\left(R_{2}, S_{2}\right)=\left(R_{1} R_{2}, S_{1}+R_{1} S_{2}\right) .
$$

The group action $\rho: S E(2) \longrightarrow G L\left(X^{\alpha}\right)$ of the group $S E(2)$ on the function space of spatial profiles $u(t, \cdot) \in X^{\alpha}$ is defined by

$$
\left(\rho_{(R, S)} u\right)(x):=u\left(R^{-1}(x-S)\right) .
$$

Thus, an element $g=(R, S)$ of $S E(2)$ simply rotates and translates the profile $u$. The seemingly strange inverses in (3) ensure, together with (2), that $\rho_{g_{1} g_{2}} u=\rho_{g_{1}} \rho_{g_{2}} u$. Given this bounded linear group action, equivariance of the vector field in equation (1) can be stated as:

$$
D \Delta\left(\rho_{g} u\right)+F\left(\rho_{g} u\right)=\rho_{g}(D \Delta u+F(u)) \quad \text { for all } g \in S E(2), u \in H^{2} .
$$

The equivariance property (4) simply means: whenever $u(t) \in X^{\alpha}$ solves reaction-diffusion system (1), then $\rho_{g} u(t) \in X^{\alpha}$ is also a solution, for any fixed choice of $g=(R, S) \in S E(2)$. In terms of the semiflow $\phi_{t}$ this property can be expressed as

$$
\phi_{t}\left(\rho_{g} u\right)=\rho_{g} \phi_{t}(u) \quad \text { for all } g \in S E(2) .
$$

Substantial mathematical difficulties arise because the group action $\rho$ is only strongly continuous. Moreover the group $S E(2)$ is non-compact due to its translational component. We suppress these technicalities in the following.

Supported by experimental and numerical evidence, we assume there exists a rigidly rotating solution of (1). In other words, there exists $u_{*} \in X^{\alpha}$ such that the time evolution of this initial condition is a pure rotation with non-zero frequency $\omega_{*}$ :

$$
\phi_{t}\left(u_{*}\right)=\rho_{\left(R_{\omega_{*}}, 0\right)} u_{*} .
$$




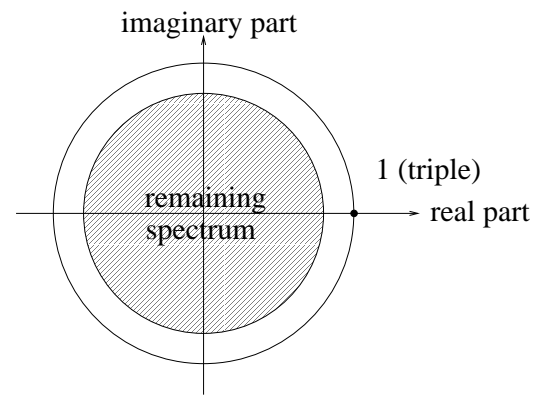

Fig. 2. Floquet multipliers $\mu$ of $D \phi_{2 \pi / \omega_{*}}\left(u_{*}\right)$ in the complex plane.

This assumption implies that $\phi_{t}\left(u_{*}\right)$ is a time-periodic solution of (not necessarily minimal) period $2 \pi / \omega_{*}$. An element $u_{*}$ of our phase space $X^{\alpha}$ is called a relative equilibrium if the time evolution $\phi_{t}\left(u_{*}\right)$ lies inside the group orbit $\rho_{g}\left(u_{*}\right)$. Hence the periodic solution of $u_{*}$ consists of relative equilibria.

Spectral analysis of the linearized semiflow along a periodic solution is called Floquet theory. The eigenvalues $\mu$ of the linearized period map are called Floquet multipliers. A Floquet exponent is a complex $\beta$ such that $\exp (\beta \tau)$ is a Floquet multiplier of the system, where $\tau$ denotes the minimal period. A periodic solution is hyperbolic if, and only if, it possesses only the trivial Floquet multiplier $\mu=1$ on the unit circle, and this multiplier has algebraic multiplicity one. Otherwise it is called non-hyperbolic. In ODEs hyperbolic periodic solutions possess stable and unstable manifolds, similarly to the case of hyperbolic equilibria. Non-hyperbolic periodic solutions possess center manifolds.

We now return to our setting (1) and assume that $\mu=1$ is a Floquet multiplier of the periodic solution $\phi_{t}\left(u_{*}\right)$, with three-dimensional generalized eigenspace. The remaining spectrum we assume to lie strictly in the interior of the complex unit circle. Due to Euclidean symmetry there exist at least three eigenvectors of $D \phi_{2 \pi / \omega_{*}}\left(u_{*}\right)$ corresponding to the eigenvalue 1, see Figure 2. The eigenvectors are the three partial derivatives $\partial_{t} u, \partial_{x_{1}} u$ and $\partial_{x_{2}} u$, of the solution $\phi_{t}\left(u_{*}\right)$, at $t=0$.

Our spectral assumption can also be expressed in a rotating coordinate frame ([Wul96], [SSW97]). Let

$$
L:=D \Delta+\omega_{*} \frac{\partial}{\partial \varphi}+D_{u} F\left(u_{*}\right),
$$

where $\frac{\partial}{\partial \varphi}$ denotes the partial derivative with respect to the angular component, i.e. $\frac{\partial}{\partial \varphi}=x_{1} \partial_{x_{2}}-x_{2} \partial_{x_{1}}$. In a co-rotating coordinate frame, the relative equilibrium $u_{*}$ becomes an equilibrium. Eigenvalues of $L$ are therefore Floquet exponents of the periodic solution $\phi_{t}\left(u_{*}\right)$. The equivalent spectral assumption is basically that zero is a triple eigenvalue of $L$ and the remaining spectrum has negative real part, uniformly bounded away from the imaginary axis. 
The symmetry of equation (1), which causes the non-hyperbolic eigenvalue zero to be triply degenerate, gives rise to a three-dimensional invariant manifold of $\phi_{t}\left(u_{*}\right)$. This is the center manifold which coincides with the group orbit of $u_{*}$, namely $S E(2) u_{*}:=\left\{\rho_{g} u_{*} ; g \in S E(2)\right\}$. Thus the center manifold of $u_{*}$ is simply the set of all translations and rotations of the initial rotating wave $u_{*}$. See [SSW97] for this result and some generalizations.

\subsection{Lattice symmetry}

The main mathematical feature of the above invariant center manifold $S E(2) u_{*}$ is its normal hyperbolicity. An invariant manifold is called normally hyperbolic if the linearized dynamics in the normal directions are of faster exponential rate than those in tangential direction. Normally hyperbolic invariant manifolds persist under small perturbations [HPS77].

To account for static, lattice periodic light patterns in the photosensitive BZ reaction, we add a symmetry breaking perturbation $\varepsilon H(u)$ to equation (1) as follows:

$$
u_{t}=D \Delta u+F(u)+\varepsilon H(u) .
$$

We assume that $\varepsilon$ is small and $H \in C^{k+2}\left(X^{\alpha}, X\right)$ keeps only a translational lattice symmetry $\mathbb{Z}^{2}$, but breaks the rotational symmetry. Therefore the semiflow $\phi_{t}^{\varepsilon}$ of the perturbed equation (8) is only lattice equivariant:

$$
\phi_{t}^{\varepsilon}\left(\rho_{(0, S)} u\right)=\rho_{(0, S)} \phi_{t}^{\varepsilon}(u) \quad \text { for all } S \in \mathbb{Z}^{2} .
$$

For example $H$ could be a superposition operator $(H(u))(x)=h(x, u(x))$, where $h: \mathbb{R}^{2} \times \mathbb{R}^{N} \rightarrow \mathbb{R}^{N}$ is a $C^{k+2}$-function that satisfies mild growth conditions. Translational equivariance then requires $h(x+S, u)=h(x, u)$ for all $S \in \mathbb{Z}^{2}$.

In the photosensitive BZ reaction this corresponds to a lattice symmetric lighting pattern of the $\mathrm{BZ}$ medium. We recall our fundamental question: Which motions of the spiral tip can be realized by choosing an appropriate perturbation $H$ ? According to equivariance condition (9) the lighting pattern in the photosensitive BZ reaction can only be chosen freely on a unit square of the underlying media. The function $H$ is then determined on the entire plane, by $\mathbb{Z}^{2}$-periodic extension. The main result below asserts, that for every prescribed ODE motion of the perturbed spiral wave tip there is a lighting pattern, represented by $H$, such that this prescribed motion can be realized, to leading order in the perturbation parameter $\varepsilon$. An example of an "illumination" functional $H$ will be given such that, both, pinning and drifting of the perturbed spiral tip, coexist.

We now state the center manifold theorem of the perturbed system:

Theorem 1 [Jan03] Assume the existence of a rotating wave solution $u_{*}$ of the unperturbed system. Suppose 1 is a triple Floquet multiplier of the linearized flow and the remaining spectrum lies strictly in the interior of the 
complex unit circle. Then, for $\varepsilon$ sufficiently small, there exists a lattice- and flow-invariant three-dimensional manifold $\mathcal{M}^{\varepsilon} \subset X^{\alpha}$. The manifold $\mathcal{M}^{\varepsilon}$ is a locally exponentially attracting manifold. Differentiability of $\mathcal{M}^{\varepsilon}$ is $C^{k, 1}$, including dependence on the parameter $\varepsilon$ : the manifold can be represented by maps with Lipschitz continuous $k$-th derivatives.

We briefly comment on the basic steps in the proof of theorem 1. For homogeneous lighting of intermediate strength a rigidly rotating spiral wave solution was assumed to be given by $u_{*}$. The manifold $\mathcal{M}^{\varepsilon}$ is close to the unperturbed normally hyperbolic center manifold $S E(2) u_{*}$ given by the translations and rotations of the spiral wave $u_{*}$. The existence of the manifold $\mathcal{M}^{\varepsilon}$ implies that the spiral shapes will stay close to that of $u_{*}$, even under slightly lattice periodic lighting patterns which destroy the full Euclidian equivariance. The symmetry breaking will cause the slightly deformed spirals to move. Projecting the dynamics of $\mathcal{M}^{\varepsilon}$ onto the 3-dimensional reference manifold $S E(2) u_{*}$ yields the dynamics of the perturbed spirals, via the rotational component $R$ and the two translational components $S$ of $S E(2)$. The rigid rotation of the unperturbed spiral $u_{*}$ is replaced by a slow modulation of the rotation frequency. The translational component becomes time dependent and is responsible for non-trivial tip dynamics.

The center manifold in theorem 1 is constructed by applying the method of the graph transform. We give a brief summary here; for further background see Shub [Shu87]. First we choose appropriate local coordinates in a neighborhood of the group orbit. The neighborhood of the group orbit $S E(2) u_{*}$ is a vector bundle over $S E(2) u_{*}$. Therefore every profile $u \in X^{\alpha}$ sufficiently close to the group orbit $S E(2) u_{*}$ can be written as the sum of an element of the group orbit $S E(2) u_{*}$ and a small element of an infinite-dimensional subspace $V$ of $X^{\alpha}$ which corresponds to the stable eigenspace of the linearized flow. See also Figure 4 below. Thus $u$ has a shape close to (a translated or rotated version of) the spiral wave solution $u_{*}$. A section of the vector bundle is a function mapping every element of $S E(2) u_{*}$ to the complementary infinite-dimensional stable eigenspace. The perturbed flow $\phi_{T}^{\varepsilon}$ applied to the graph of a section will again be a section of the bundle due to normal hyperbolicity of $S E(2) u_{*}$ if $T$ is large enough. This graph transform, which maps sections to sections, becomes a contraction on the space of Lipschitz-continuous sections equipped with the metric induced by the sup-norm. Hence it has a unique fixed point and the graph of the fixed point is the perturbed center manifold $\mathcal{M}^{\varepsilon}$.

Using graph transforms, $\mathcal{M}^{\varepsilon}$ automatically becomes time- and flow-invariant. Smoothness of the manifold $\mathcal{M}^{\varepsilon}$ is a very delicate question and has been settled in [Jan03].

As an example we state the two-component Oregonator model of the photosensitive BZ reaction with external forcing, see for example [ $\left.\mathrm{ZBB}^{+} 04\right]$ : 


$$
\begin{aligned}
\frac{\mathrm{d} u}{\mathrm{~d} t} & =\Delta u+\frac{1}{\varepsilon}\left[u-u^{2}-(f v+I(t)) \frac{u-q}{u+q}\right], \\
\frac{\mathrm{d} v}{\mathrm{~d} t} & =u-v .
\end{aligned}
$$

Here $u$ corresponds to the concentration of the autocatalytic species, bromous acid $\mathrm{HBrO}_{2}$, and $v$ corresponds to the oxidized form of the catalyst. The parameters $\varepsilon=0.05, q=0.002$, and $f=2.0$ are fixed and $I=I(t)$, induced by external light intensity, describes the bromide production.

\subsection{Spiral tip dynamics}

Many definitions of spiral tips are in use. One of them considers the spiral tip as "the" point of the spiral wave front with maximal curvature [BE93a]. A practical way to determine the tip position in an experiment is to look at spiral wave fronts extracted from two consecutive frames of a digitized recording. The intersection of these lines is also considered a tip position [KGZM01]. For several other definitions see [Zyk87, JSW89] and Figure 3.

In this section we clarify the mathematical relation - and equivalence of all this zoo of tip definitions; see (11)-(21) below. The main point is that any tip function $z^{*}: X^{\alpha} \rightarrow \mathbb{C}$ should associate a tip position $z^{*}(u)$ to a spatial profile $u \in X^{\alpha}$, such that shifted or rotated profiles $u$ give rise to a correspondingly shifted and rotated tip position $z^{*}$. Any reasonable definition of a tip function $z^{*}$ will certainly have to satisfy at least this minimal condition of equivariance with respect to the Euclidean group $S E(2)$. It turns out, below, that any tip definition then gives rise to the same basic form of the reduced

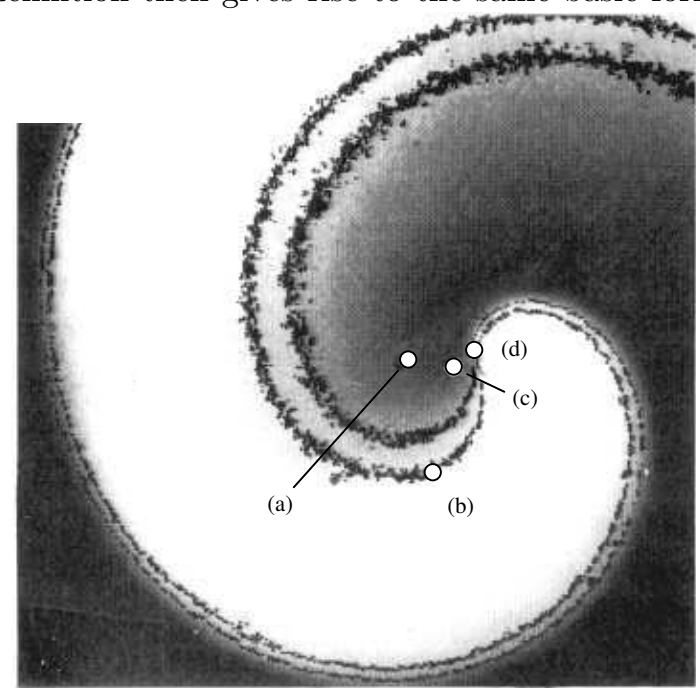

Fig. 3. "Tips" of spiral wave pattern in the BZ reaction: a) core center, b) maximum curvature, c) rotation center, d) inflection point; see [MZ94]. 
ODE on a center manifold near the unperturbed reference spiral wave shape $S E(2) u_{*}$, as long as the tip faithfully represents the translation action in $S E(2)$.

We discuss the interpretation of the tip function $z_{*}$ for the spatially homogeneous, fully $S E(2)$-equivariant case first. The modifications for the photosensitive case with lattice symmetry will be discussed in section 2.5 below.

From [SSW97] we recall the existence of a center manifold $\mathcal{M}$ associated to a rigidly rotating reference spiral, alias a relative equilibrium $S E(2) u_{*}$; see also (1)-(7) above. In fact, the center manifold $\mathcal{M}$ accounts for all solutions which remain in a neighborhood of the relative equilibrium $S E(2) u_{*}$ for all positive and negative times. In particular, all bifurcations due to point spectrum on the imaginary axis of the linearization $L$ from (7) occur in the finite-dimensional center manifold $\mathcal{M}$. For simplicity of presentation, however, we then suppress the dependence of $\mathcal{M}$, and of the flow on $\mathcal{M}$, on any extra bifurcation parameters.

To perform any specific calculations, and to clarify the zoo of possible tip functions $z^{*}$, we need good coordinates on $\mathcal{M}$ : coordinate free abstract nonsense will not suffice here. Palais has constructed good coordinates near group orbits of proper, but not necessarily compact, group actions. We apply these coordinates to the group $S E(2)$ and the reference group orbit $S E(2) u_{*}$ of rigidly rotating waves. Let $g \in S E(2)$ and let $v \in V$ denote elements of a local section $\mathrm{V}$, transverse to the group orbit $S E(2) u_{*}$ in the center manifold $\mathcal{M}$. Clearly $g v$, or more precisely $\rho_{g} v$, then cover a tubular neighborhood of $S E(2) u_{*}$, see Figure 4 . Notationally, we may let $v=0$ correspond to $u_{*}$ itself here.

In [FSSW96] the resulting ODEs in the center manifold $\mathcal{M}$ of a relative equilibrium $S E(2) u_{*}$ were derived, in these Palais coordinates. Suppressing extra parameters, again, the result is

$$
\begin{aligned}
& \dot{g}=a(v) g \\
& \dot{v}=\varphi(v) .
\end{aligned}
$$

Here $a: V \rightarrow \operatorname{alg}(S E(2))$ is a function from the section $V$ to the Lie algebra of $S E(2)$, and $\varphi(v)$ denotes a vector field on $V$. Anyway, please read on. To be more specific, we rewrite (11) in terms of suitable coordinates $g=(R, S)$ on the Euclidean group $S E(2)$. Using complex notation, we write $g=(R, S)=$ $\left(e^{i \alpha}, z\right)$. Here $R=e^{i \alpha}$, acting multiplicatively, denotes rotations of $x \in \mathbb{R}^{2} \cong$ $\mathbb{C}$. The translation component $S=z \in \mathbb{R}^{2} \cong \mathbb{C}$, acting additively, denotes translations. In coordinates $(\alpha, z, v) \in(\mathbb{R} / 2 \pi \mathbb{Z}) \times \mathbb{C} \times V$, system (11) now demystifies and reads

$$
\begin{aligned}
\dot{\alpha} & =\omega(v) \\
\dot{z} & =e^{i \alpha} \sigma(v) \\
\dot{v} & =\varphi(v) .
\end{aligned}
$$

with given functions $(\omega, \sigma): V \rightarrow \mathbb{R} \times \mathbb{C}$. 


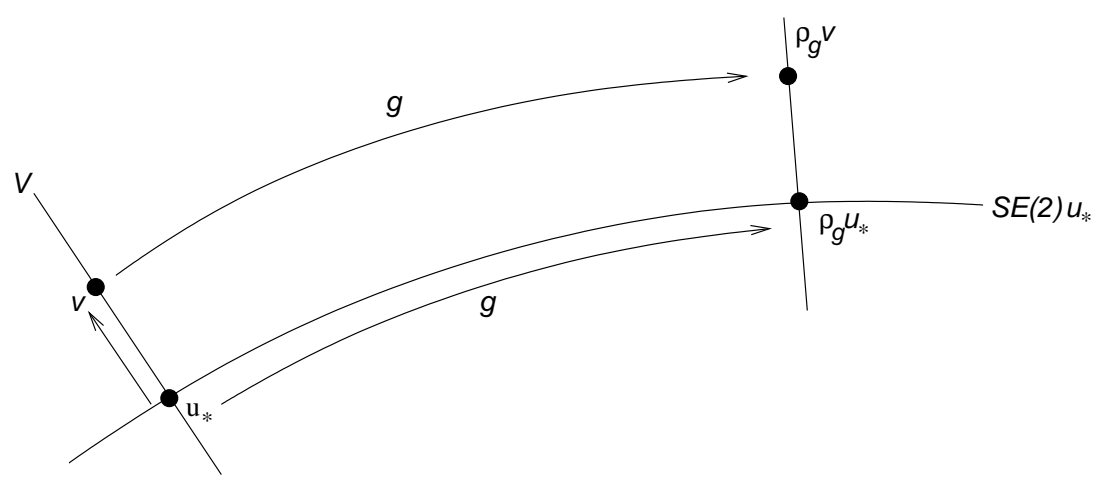

Fig. 4. The construction of local Palais coordinates $(g, v)$ for a tubular neighborhood of a group orbit $S E(2) u_{*}$.

How to interpret systems (11), (12)? First of all, let us interpret the Palais coordinates $(g, v)=\left(e^{i \alpha}, z, v\right) \in S E(2) \times V$ themselves. Obviously, the definition of Palais coordinates implies that elements $u_{1}, u_{2}$ in the same group orbit have the same component $v$. Therefore $v$ parametrizes the shape of spatial profiles, in our setting. Indeed, spatial profiles $u_{1}, u_{2} \in X^{\alpha}$ which have the same $v$-component differ only in $g$, viz. by a rotation and translation of their spatial profiles:

$$
u_{2}(x)=\left(\rho_{g} u_{1}\right)(x)=u_{1}\left(e^{-i \alpha}(x-z)\right) .
$$

The group coordinates $g=\left(e^{i \alpha}, z\right)$ then correspond to a translation of the spatial profile by $z$, and a rotation by an angle $\alpha \in \mathbb{R} / 2 \pi \mathbb{Z}$.

In Palais coordinates $(g, v)=\left(e^{i \alpha}, z, v\right)$, systems (11), (12) have skew product structure: the pure shape dynamics $\dot{v}=\varphi(v)$ of the shape variable $v$ is autonomous, and is not influenced by the group coordinates $g=\left(e^{i \alpha}, z\right)$ of rotation angle $\alpha$ and (complex) translation $z$. On the other hand, the shape variable $v$ acts as a forcing on the group variables $\left(e^{i \alpha}, z\right)$.

We are now ready to clarify the role of tip functions $z^{*}: \mathcal{M} \rightarrow \mathbb{R}^{2} \cong \mathbb{C}$. We only assume equivariance

$$
z^{*}\left(\rho_{g}(u)\right)=g z^{*}(u)
$$

for any $g \in S E(2), u \in \mathcal{M}$. In fact we reduce attention to the center manifold $\mathcal{M}$ because all bifurcation phenomena must occur in $\mathcal{M}$. We claim that we may substitute $z^{*}=z^{*}(u)$ for the translation component $z$ in the Palais coordinates $\left(e^{i \alpha}, z, v\right)$, without changing the skew product structure of the ODE (12) on the center manifold $\mathcal{M}$.

Indeed, equivariance condition (14) on the tip function implies that

$$
z^{*}=z^{*}(u)=z^{*}\left(\rho_{\left(e^{i \alpha}, z\right)} v\right)=\left(e^{i \alpha}, z\right) z^{*}(v)=e^{i \alpha} z^{*}(v)+z .
$$

This defines a transformation 


$$
\left(e^{i \alpha}, z, v\right) \mapsto\left(e^{i \alpha}, z^{*}, v\right)
$$

with inverse given explicitly by

$$
z=z^{*}-e^{i \alpha} z^{*}(v)
$$

Thus the tip position $z^{*}$ may indeed be considered as the translation component $z$ of the Palais coordinates, directly, up to a coordinate transformation (16). Let us calculate, briefly, the resulting skew product ODE component for $z^{*}$ :

$$
\begin{aligned}
\frac{\mathrm{d}}{\mathrm{d} t} z^{*} & =\frac{\mathrm{d}}{\mathrm{d} t}\left(e^{i \alpha} z^{*}(v)+z\right)=i \dot{\alpha} e^{i \alpha} z^{*}(v)+e^{i \alpha} z^{* \prime}(v) \dot{v}+\dot{z}= \\
& =e^{i \alpha}\left(i \omega(v) z^{*}(v)+z^{* \prime}(v) \varphi(v)+\sigma(v)\right)=: e^{i \alpha} \sigma^{*}(v)
\end{aligned}
$$

with the obvious definition for the new function $\sigma^{*}(v)$. Therefore the tip function $z^{*}$ satisfies a transformed ODE of the same structure as the translation component $z$ itself.

For illustration purposes, let us now interpret meandering and drift in terms of the Palais coordinates $\left(e^{i \alpha}, z, v\right)$. First note that the relative equilibrium $v=0$, which corresponds to the rigidly rotating spiral, becomes an equilibrium $\varphi(0)=0$ of the shape ODE $\dot{v}=\varphi(v)$ in (12). Indeed the group orbit $S E(2) u_{*}$, which corresponds to one and the same shape variable $v=0$, contains the time orbit of the rigidly rotating spiral $u_{*}$. Therefore

$$
\begin{aligned}
\dot{\alpha} & =\omega(0) \\
\dot{z} & =e^{i(\omega(0) t+\alpha(0))} \sigma(0)
\end{aligned}
$$

indicates a rigid rotation frequency $\omega_{*}=\omega(0)$ and a circular tip motion $z(t)$; see Figure 1.

Now assume the shape dynamics $\dot{v}=\varphi(v)$ undergoes a Hopf bifurcation from this trivial (relative) equilibrium $v=0$, albeit with suppressed parameters. Then $v(t)$ will be periodic, say with "breathing" frequency $\omega_{1}$ of the pure shape dynamics. Multiplying (12) by the nonzero Euler multiplier $\omega_{*} / \omega(v) \approx 1$ and redefining $\sigma(t):=\sigma(v(t)) \omega_{*} / \omega(v(t))$ we obtain the tip equation

$$
\dot{z}=e^{i \alpha(t)} \sigma(t)=e^{i \omega_{*} t} \sigma(t)
$$

Obviously, $\sigma(t)$ has inherited minimal period $2 \pi / \omega_{1}$ from the shape equation. Fourier expansion of $\sigma(t)$ and direct integration of (20) implies that $z(t)$ undergoes a 2-frequency bounded meandering epicycle motion, unless the frequencies

$$
\omega_{*}=m \omega_{1}
$$

are in integer resonance, for some $m \in \mathbb{N}$. In the latter case, nonvanishing $(-m)$-th complex Fourier coefficients of $\sigma(t)$ will provide an unbounded drifting motion which is linear in $t$. Note that the rotation frequency $\omega_{*}$ must be 
an integer multiple of the breathing frequency $\omega_{1}$ of the pure shape dynamics $\dot{v}=\varphi(v)$, for drifting tip motion to occur. Both, meandering and drifting spirals change their shape $v(t)$ periodically and are therefore also called relative periodic orbits.

By our above considerations, these meandering/drifting effects can be interpreted as tip motions, directly. The results are universal, at the same time, in the sense that the observed motions do not depend on the particular choice of a tip function $z^{*}(u)$ from the large zoo of $S E(2)$-equivariant possibilities.

Nonautonomous time-periodic forcings with frequency $\omega_{1}$ can be treated analogously. Indeed, $\omega=\omega(t)$ and $\sigma=\sigma(t)$ then depend on time $t$ directly and our remarks on (20), (21) apply. We will return to this observation when we describe the alternative kinematic approach, in section 3.

Some arbitrariness is still involved in the choice of the transverse Palais section $V$ in Figure 4. This geometric arbitrariness can in fact be used to further simplify the skew product form (11). Such an approach, which first simplifies $\dot{v}=\varphi(v)$ to Poincaré-Birkhoff normal form, and then redefines $V$ to further simplify the cross term $a(v)$, has been pursued in [FT98].

\subsection{Reduced tip equations for the photosensitive system}

As above, we consider an unperturbed reference spiral wave $S E(2) u_{*}$ which is rigidly rotating with constant frequency $\omega_{*}$. In this section we adapt the considerations of section 2.4 on the tip dynamics $z^{*}(t)$, and the skew product flow (12) in the center manifold $\mathcal{M}$, to lattice periodic perturbations $\varepsilon H(u)$; see (8). We recall that such perturbations account for slight, spatially periodic but temporally constant, variations of light intensity in the photosensitive BZ reaction.

Under the spectral assumptions of section 2.3, theorem 1, it is now possible to reduce the perturbed dynamics to a three-dimensional center manifold $\mathcal{M}^{\varepsilon}$ which is modeled over the group $S E(2)$ itself. In Palais coordinates

$$
\begin{aligned}
\dot{\alpha} & =\omega_{*}+\varepsilon \gamma(\alpha, z, \varepsilon) \\
\dot{z} & =\varepsilon h(\alpha, z, \varepsilon) .
\end{aligned}
$$

As was justified in section 2.4, the angle $\alpha$ denotes the phase and $z$ the position of the spiral tip. The Palais section coordinate $v \in V$ is absent here, because the critical spectrum is now three-dimensional, only, and is accounted for by the three-dimensional group $S E(2)$ itself. Therefore the center manifold $\mathcal{M}^{\varepsilon}$ is a graph over the group coordinates $\left(e^{i \alpha}, z\right) \in S E(2)$. A rigorous derivation of the reduced equation (22) has indeed been achieved in [Geo03, Jan03], under the assumption that the unperturbed spiral wave $u_{*}(\cdot)$ is spectrally stable with the exception of a triple critical eigenvalue due to symmetry; see theorem 1. Note that the nonlinearities $\gamma(\alpha, z, \varepsilon)$ and $h(\alpha, z, \varepsilon)$ obey the lattice symmetry relic of full Euclidean symmetry, namely 


$$
\begin{aligned}
& \gamma(\alpha, z+k, \varepsilon)=\gamma(\alpha, z, \varepsilon) \\
& h(\alpha, z+k, \varepsilon)=h(\alpha, z, \varepsilon)
\end{aligned}
$$

for every angle $\alpha \in S^{1}, z \in \mathbb{C} \cong \mathbb{R}^{2}$ and integer $k \in \mathbb{Z}^{2} \subseteq \mathbb{R}^{2}$. See also (9).

In fact (22) therefore induces a (nonautonomous) flow on the 2-torus $z \in \mathbb{C} / \mathbb{Z}^{2}$, by reinterpreting the angle $\alpha$ as the new "time". This is possible because $\dot{\alpha}(t) \neq 0$ for $\varepsilon$ sufficiently close to zero and all real $t$. Multiplying (22) by the nonzero Euler multiplier $1 /\left(\omega_{*}+\varepsilon \gamma(\alpha, z, \varepsilon)\right)$ therefore provides the nonautonomous tip equation

$$
\dot{z}=\varepsilon \widetilde{h}(\alpha, z, \varepsilon)
$$

Here we have replaced $h /\left(\omega_{*}+\varepsilon \gamma\right)$ by a new function $\widetilde{h}$, and we can consider $\alpha$ as new time because $\dot{\alpha}=1$. The lattice symmetry (9), (23) is inherited by the new nonlinearity $\widetilde{h}$. Therefore (24) indeed defines a nonautonomous slow flow on the 2 -torus $z \in T^{2}=\mathbb{R}^{2} / \mathbb{Z}^{2}$. Passing to slow time $t=\varepsilon \alpha,(24)$ transforms to the rapidly periodically forced system $\dot{z}=\widetilde{h}(t / \varepsilon, z, \varepsilon)$, where $h$ is $2 \pi$-periodic in its first argument $\alpha=t / \varepsilon$. Standard averaging procedures then reduce (24) to the autonomous system

$$
\dot{z}=h(z, \varepsilon),
$$

up to any finite order in $\varepsilon$, with a suitably defined new vector field $h$, [SV85]. For example,

$$
h(z, 0)=\frac{1}{2 \pi} \int_{0}^{2 \pi} \widetilde{h}(\alpha, z, 0) \mathrm{d} \alpha
$$

turns out to simply be the average of the nonautonomous vector field $\widetilde{h}$ over its fast periodic forcing variable $\alpha=t / \varepsilon$ - hence the name averaging for this procedure. For more advanced results on averaging see for example [FS96] or [GL01, Gel02] and the references there.

As expected, one easily recovers the unperturbed spiral tip motion

$$
\dot{z}(t)=0
$$

in equation (24), by setting $\varepsilon=0$. Of course, the precise form of $\widetilde{h}$ and $h$ in (24) and (22) depends crucially on the exact choice of the original PDE perturbation $H$. We write $h=h(z, \varepsilon ; H)$ in $(22)$ to emphasize this important dependence. In particular, the choice of $H$ determines the vector field (22) which itself determines the (averaged) spiral tip dynamics (25).

Expanding $h(z, \varepsilon)$ with respect to small $\varepsilon$ yields

$$
h(z, \varepsilon ; H)=h_{1}(z ; H)+\varepsilon h_{2}(z ; H)+\ldots
$$

We study the dependence of $h_{1}$ on $H$, because $h_{1}$ governs the dynamics (25) of the perturbed spiral wave tip, to leading order in $\varepsilon$. Therefore we consider the map 


$$
\mathcal{L}: H \mapsto h_{1}(H)
$$

where $h_{1}(H)=h_{1}(\cdot, H)$. For the perturbation $H$ we choose any bounded $C^{k+2}$-functional which satisfies the lattice symmetry. Then $\mathcal{L}$ maps $H$ into the space of all vector fields $h$ on $\mathbb{R}^{2}$ of class $C^{k}$ which satisfy

$$
h(z+k)=h(z),
$$

for every $z \in \mathbb{C} \cong \mathbb{R}^{2}$ and $k \in \mathbb{Z}^{2}$.

The range of $\mathcal{L}$ has an interesting interpretation: It represents the vector fields of all possible motions of the perturbed spiral wave tip $z(t)$, to leading order in $\varepsilon$. Indeed, a vector field $h$ in the range of $\mathcal{L}$ allows one to find a function $\mathrm{H}$ with $h=h_{1}(H)$ and thus determines the motion of the spiral tip to leading order; see (22).

The main result now states that the mapping $\mathcal{L}$ is linear, continuous and surjective; see [Geo03, GJ05]. Note that this result does not depend on the particular coordinates which have to be introduced in order to obtain the reduced averaged equation (25). Continuity and linear dependence of $\mathcal{L}$ on the function $H$ is very intuitive: By slightly changing the light intensity pattern one expects the motion of the spiral wave to change only slightly. Also a superposition of two different light intensity patterns $H_{1}$ and $H_{2}$ should result in a superposition of the corresponding motions of the spiral tip positions, at least to leading order. Although the surjectivity of $\mathcal{L}$ may sound just as reasonable, from a physical point of view, we have to keep in mind that $h=$ $h_{1}(H)$ for some $H$ is the leading order term which governs the time averaged dynamics of the perturbed spiral tip, see (28). From a mathematical point of view it could happen, that certain changes in the light intensity pattern, and thus in $H$, influence the spiral tip motion only in the second order terms of (28) with respect to $\varepsilon$. Moreover, local perturbations $H(u)(x)=H(x, u(x))$ which merely evaluate the pattern $u(x)$ at any given point $x$, may not suffice to ensure surjectivity of $\mathcal{L}$. Within the larger class of functionals $H$, rather than just functions, however, the strong property of surjectivity prevails.

We repeat that the construction of the functional $H$ in [Geo03] involves quantities such as the group orbit $S E(2) u_{*}$ of the unperturbed spiral wave. In particular, $H$ will be a nonlocal functional, in general. Keeping in mind that the experimentalist has only a few control parameters at hand, it remains a challenging task to adjust the light intensity pattern in order to obtain the desired spiral tip motion. For specific results in this direction see $\left[\mathrm{ZBB}^{+} 04\right.$, ZE04, ZBBE05, KM05, ZE06], and our brief discussion in section 4.

Up to these difficulties, we have seen that there is a certain lattice periodic perturbation pattern, represented by a functional $H$, which realizes a prescribed averaged tip motion (25).

\subsection{Pinning versus drifting}

It was shown in the previous section that every vector field of the perturbed spiral tip motion can be realized, to leading order, by choosing an appropri- 
(a)

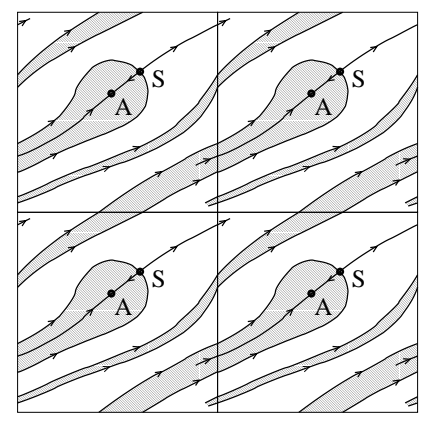

(b)

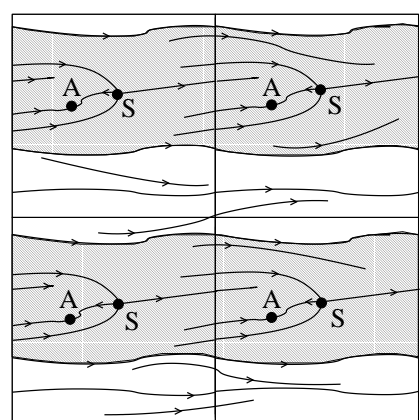

Fig. 5. Cherry flows: (a) irrational winding; (b) rational winding with stable and unstable periodic drifts. Basins of $A=$ pinning (grey), and drifting region (white).

ate pattern which is represented by a lattice periodic, nonlinear perturbation functional $H$. We recall from (25) that the motion of the perturbed spiral wave tip is governed by the averaged differential equation

$$
\dot{z}=h(z, \varepsilon)
$$

on the 2-torus $z \in \mathbb{C} / \mathbb{Z}^{2}$

We now consider a particular such vector field, where pinning of the perturbed spiral tip occurs, and coexists with drifting motions. This particular flow on the torus is called the Cherry flow, see Figure 5(a) and, for further background, [PdM82]. The Cherry flow features two equilibria, a saddle $S$ and a sink $A$. Every solution $z(t)$ with initial tip position $z(0)$ in the open grey basin gets attracted to the $\operatorname{sink} A$, eventually. The stable manifold $W^{s}(S)$ of the saddle $S$ marks the boundary of the basin of attraction of $A$ and denotes points which converge to $S$, eventually. For other initial values (white region) the solution neither gets attracted to the saddle $S$ nor to the sink: the spiral keeps drifting.

We state an explicit example of such a vector field for $z=\left(z_{1}, z_{2}\right)$ :

$$
\begin{aligned}
& \dot{z}_{1}=c_{1}-\sin \left(2 \pi z_{1}\right)+c_{2}\left(1-\cos \left(2 \pi z_{2}\right)\right) \\
& \dot{z}_{2}=-\sin \left(2 \pi z_{2}\right)
\end{aligned}
$$

with constants $0<c_{1}<1$ and $c_{2} \neq 0$. Because the vector field (32) is 1 -periodic in $z_{1}, z_{2}$, it indeed defines a vector field on the 2 -torus.

Keeping in mind that the dynamics of (31) represents the motion of the perturbed spiral wave tip, this example shows that there are indeed open sets of initial positions $z(0)$ for which the spiral tip $z(t)$ finally gets pinned to the $\operatorname{sink} A$. Coexisting are regions for which the spiral tip never gets attracted to any specific point and undergoes a drifting motion on the 2-torus, which is unbounded in $\mathbb{R}^{2}$. The regions are interwoven in a Cantor-like structure. It should be mentioned that the drifting region has zero Lebesgue measure in the above example; see also [Men91, MR92]. Zero Lebesgue measure implies that 
almost every spiral tip eventually becomes pinned, but possibly after a very long transient phase of drifting. In general, however, it seems that drifting can also prevail on regions of positive Lebesgue measure.

At least in presence of periodic orbits on the 2-torus, drifting and pinning can coexist with positive measure; see Figure 5(b). Indeed the pinning region of the attracting equilibrium (A) is now trapped between two unstable periodic orbits. The unstable periodic orbits also bound the basin of attraction of a stable periodic orbit. The stable periodic orbit signifies stable unbounded drift of the spiral tip across the lattice periodic perturbation pattern.

\section{Kinematics}

\subsection{Curves and tips}

The kinematic approach to the propagation of planar excitation waves idealizes the wave front location to be given by a one-dimensional curve

$$
z=Z(t, s) \in \mathbb{R}^{2} \cong \mathbb{C} .
$$

The curve $Z$ is parametrized by arc length $s$ at any given time $t$. The basic modeling assumption is then the hypothesis that the curve $Z(t, s)$ moves in normal direction $\underline{n}:=i Z_{s}$ with a normal velocity $U \in \mathbb{R}$ which depends on the curvature

$$
\kappa \underline{n}:=-Z_{s s} .
$$

We abbreviate this modeling assumption as

$$
U=U(t, \kappa),
$$

where the explicit dependence on $t$ allows for external forcing. We will obtain rigidly rotating curves as well as meanders and drifts in this setting.

In the mathematical literature, the special case of a power law dependence

$$
U=U(\kappa)=-\kappa^{\beta},
$$

with $\beta, \kappa>0$ has attracted much attention under the name of "curve shortening"; see for example [GH86, Ang90, Ang91, FM02] and the references there. Closed convex curves $Z$, for example, shrink to a point in finite time, for $\beta=1$, and become asymptotically circular before they disappear. A celebrated generalization of this fact to convex surfaces under mean curvature flow was obtained by [Hui93]. Much excitement has been caused in the mathematical community, recently, by Perelman's contributions relating flows of compact three-dimensional manifolds under their Ricci curvature to the Poincaré conjecture [Per06a, Per06b, Per06c].

Due to their parabolic PDE nature, curvature flows have a smoothing effect on the curves $Z(t, \cdot)$. Curvature flows are therefore also used in image 
processing to smoothen ragged boundaries [MS95, CM01, Mik01]. Of particular interest in image processing is the case $\beta=1 / 3$, which is in addition affine equivariant: initial curves which are affine images of each other retain this property under their evolution (36).

As we have noted in the introduction, experimental evidence for a kinematic description of excitable wave fronts is rich. Based on hyperbolic wave equations and the Huygens principle, [WR46] recommend the eikonal approach of geometric optics: waves propagate at a constant normal speed

$$
U(t, \kappa)=c(t),
$$

which does not depend on the curvature of the wave front. In section 3.3 below, we will describe meander and drift of spirals under this very restrictive assumption. More general affine linear dependencies

$$
U(t, \kappa)=c(t)-D(t) \kappa,
$$

albeit with constant coefficients $c, D$, have been proposed by several authors, both for BZ systems ([TK88, MZ91]) and for surface waves in catalysis ([IIY98, Mer92]). Motivated by the photosensitive BZ reaction we modify these models to include time dependent coefficients $c(t), D(t)$ which account for external forcing.

In section 2 we have seen how rigid rotations, meanders, and drifts are closely tied to equivariance with respect to the Euclidean group $S E(2)$ of planar rotations and translations. We therefore prefer to not describe our curvature flows in terms of the position vector $Z(t, s) \in \mathbb{C}$, directly. Instead, we work with the curvature scalar

$$
\kappa=\kappa(t, s),
$$

which eliminates the $S E(2)$-action. Indeed, curves $Z_{1}(t, s)$ and $Z_{2}(t, s)$ are Euclidean images of one another, under some fixed $(R, S) \in S E(2)$, if and only if their curvature functions $\kappa$ coincide. Therefore the parametrization by $\kappa$ can be viewed as a particularly suitable choice of a Palais shape coordinate $v$, in the terminology of section 2.4; see (11), (12) and Figure 4.

An important modification to the standard approaches concerns our treatment of the spiral tip. Traditionally, waves in excitable media are thought of as arising from a singular perturbation cycle in the reaction term. This view point readily identifies a "front" and "back" for the wave. Serious difficulties obstruct joining these curves in the sense of matched asymptotic expansions [Kee92]. Instead, we merge "front" and "back" to be represented by a single curve. In other words, we view the excitation excursion as a single narrow pulse phenomenon, rather than a widely separated succession of first a front and then a back transition. The problematic core junction of front and back is then distilled into a single point, say $s=s_{0}(t)$, where the pulse curve $z=Z(t, s)$ terminates, or rather initiates. The above curvature flow then accounts for the dynamics of $Z(t, s)$, for $s \geq s_{0}(t)$. It is natural to call the end 
point $s=s_{0}(t)$ of the curve its tip. Certainly, such a definition is compatible with our discussion of tip choices in section 2.4. Moreover, the tip location can be seen as a degeneracy point of the singular perturbation excitable cycle, see [FM00] or, in the terminology of Winfree [Win01], as a phase singularity.

But what is the proper dynamics of the tip itself? Based on experimental evidence, we make the following three modeling assumptions:

(T1) the normal velocity of the tip is given by $U(t, \kappa)$, as everywhere else;

(T2) the tangential velocity of the tip is given by a function $G(t)$;

(T3) the curvature at the tip is given by a function $\kappa_{0}(t)>0$.

Here (T1) is just a continuity assumption. Assumptions (T2), (T3) are based on observations of initial conditions which prepare an excitable cycle across an interval, in the $x$-plane, which terminates at two endpoints. The interval then propagates, while the two end points extend and curl inward to produce a pair of spiral-antispiral cores. See [RGS ${ }^{+}$96, ZE00, BBSE00]. Careful examination of such experiments should reveal the values of $G$ and $\kappa_{0}$, quantitatively. For a theoretical attempt at a derivation of tangential speeds $G(t)$ of spiral tips see also [Pis06]. Although the more general case $G=G(t, \kappa)$ could be incorporated, we assume $G, \kappa_{0}$ to depend on $t$, only, for simplicity and for lack of experimental detail and mathematical derivation, alike.

Summarizing our discussion we arrive at the following PDE description of the dynamics of excitable media waves:

$$
\kappa_{t}+U(t, \kappa)_{s s}+\left(\kappa \int_{0}^{s} \kappa U(t, \kappa) \mathrm{d} \sigma\right)_{s}+G(t) \kappa_{s}=0
$$

for $s \geq 0$, with the boundary condition

$$
\kappa=\kappa_{0}(t) \quad \text { at } s=0 .
$$

Here the Dirichlet boundary condition (41) follows from modeling assumption (T3). The drift term $G \kappa_{s}$ in (40) arises from (T2) when we normalize the tip to occur at $s=0$ instead of $s_{0}(t)$. Likewise the integral term accounts for reparametrization by arc length, which is necessary as the propagating curve extends or contracts. See [Mer92, MDZ94, BT96] for detailed derivations.

In section 3.2 we discuss rigidly rotating wave solutions for autonomous velocity functions $U=U(\kappa)$; see also [MDZ94, IIY98] for earlier partial analysis of the affine case $U(\kappa)=c-D \kappa$. In section 3.3 we discuss the $\kappa$-independent, but forced, eikonal case $U=U(t)=c(t)$ to obtain meanders, drifts and superspiral patterns in the kinematic setting.

\subsection{Rigidly rotating spirals}

In this section we discuss stationary solutions $\kappa=\kappa(s)$ of the curvature flow (35) for autonomous normal velocity functions $U=U(\kappa)$ and constant $\kappa_{0}>0$, 
$G \neq 0$. More precisely we solve (40), (41) for relative equilibria, alias rotating waves $\kappa=\kappa(s)$, in the integrated form

$$
\begin{aligned}
U(\kappa)_{s}+\kappa \int_{0}^{s} \kappa U(\kappa) \mathrm{d} \sigma+G \kappa & =\omega \\
\kappa(0) & =\kappa_{0}
\end{aligned}
$$

with some suitable real integration constant $\omega$. In (60), (61) below, we will see that $\omega$ is in fact the rotation frequency of the rotating Archimedean spiral given by the solution $\kappa(s)$ of (42). Differentiating (42) with respect to arc length $s$ and using (42) itself to eliminate the integral term, we obtain the $G$-independent equation

$$
U(\kappa)_{s s}+\frac{\kappa_{s}}{\kappa}\left(\omega-U(\kappa)_{s}\right)+\kappa^{2} U=0
$$

with parameter $\omega$.

Our discussion of (43) follows the global and rather complete existence, multiplicity, and bifurcation analysis in [FGT04, FGT06]. This analysis assumes $U^{\prime}(\kappa) \neq 0$ and invertibility of the relation $U=U(\kappa)$ between normal velocity and curvature by an inverse function

$$
\kappa=\Gamma(U) .
$$

Mimicking the affine case $U(\kappa)=c-D \kappa$, for which $\Gamma(U)=(c-U) / D$, we assume $\Gamma \in C^{4}$ satisfies $\Gamma^{\prime}(U)<0$ for all $U$, and $\Gamma(c)=0$ for some $c>0$. This allows us to rewrite (43) as a second order equation of Lienard type,

$$
U_{s s}+\frac{\Gamma_{U}}{\Gamma} U_{s}\left(\omega-U_{s}\right)+\Gamma^{2} U=0,
$$

with singular behavior at $U=c$ where $\Gamma(U)=0$. The eikonal case $U(\kappa) \equiv c$, where such an inversion is not possible, will be addressed in section 3.3.

Our alert reader will have noticed how we "forgot" the Dirichlet boundary condition $\kappa(0)=\kappa_{0}$ in passing from (42) to (45). In fact we will supplement (45) by the Neumann boundary condition

$$
U_{s}(0)=\omega-G \kappa_{0},
$$

which readily follows from (42). We now give a bifurcation result for the special case $G=0$; see [FGT06, Theorem 1.1] in terms of the bifurcation parameter $\omega>0$ in (45), (46). We comment on the general case of nonzero $G$ and the translation to the original problem (42) at the end of this section.

Theorem 2 [FGT06, center manifold] Under the above assumptions on the normal velocity $U=U(\kappa)$ as a function of curvature $\kappa$, all solutions $U=U(s)$ of (45), (46) with bounded normal velocity $U$ and nowhere vanishing curvature, 
$\boldsymbol{U}(0)$

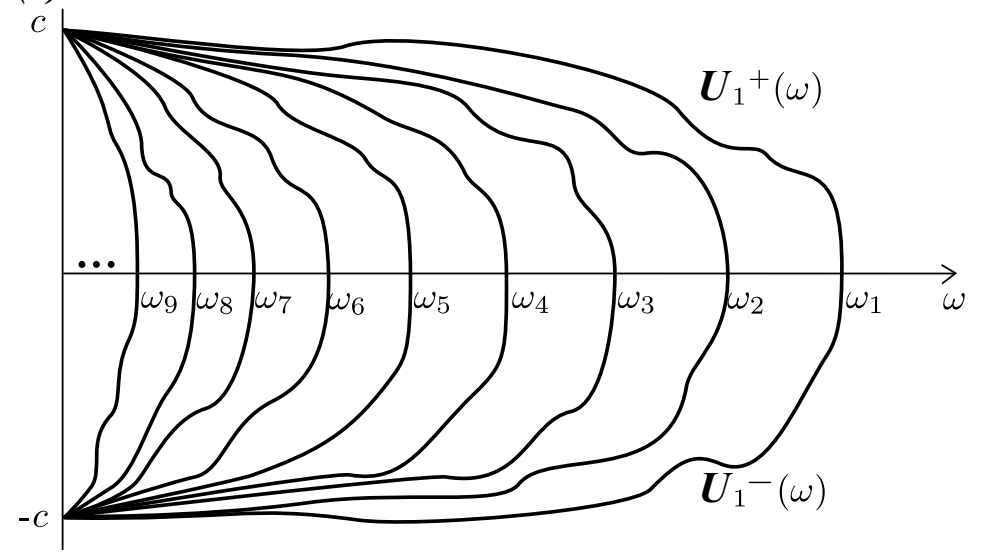

Fig. 6. A global bifurcation diagram of rotating Archimedean spirals with rotation frequency $\omega>0$ and normal tip velocity $U(0)$.

for all values of the rotation frequency $\omega>0$, are contained in the bifurcation diagram of Figure 6.

More precisely, there exists a strictly decreasing sequence $\omega_{1}>\omega_{2}>\cdots \searrow$ 0 and associated functions $U_{n}^{ \pm}(\omega)$, for $0<\omega \leq \omega_{n}$, with the following property. $A C^{3}$-solution $U=U(s), s \geq 0$ of the curvature equation (45), (46) as above exists if, and only if, the tip velocity $U(0)$ and the derivative $U_{s}(0)$ at the tip $s=0$ satisfy

$$
U(0) \in\left\{U_{n}^{-}(\omega), U_{n}^{+}(\omega)\right\}, \quad U_{s}(0)=\omega
$$

for some positive integer $n$.

The associated curves $z=Z(t, s)=r \exp (i \varphi)$ are right winding, left rotating spirals which are asymptotically Archimedean, for $s \rightarrow \infty$. The tip $Z(t, 0)$ rotates on a circle of radius $\rho=|U(0) / \omega|$. The asymptotic wave length of the Archimedean spiral in the far field $r \rightarrow \infty$ is given by $2 \pi c / \omega$.

The functions $\omega \mapsto U_{n}^{ \pm}(\omega)$ possess the following properties, for every $n \in \mathbb{N}$ :

(i) the union of the graphs of $U_{n}^{ \pm}$forms a $C^{3}$-curve, with nonvanishing curvature at $\omega=\omega_{n}$

(ii) $U_{n}^{ \pm}(\omega)=0$, and $U_{n}^{ \pm}(\omega) \rightarrow \pm c$ for $\omega \searrow 0$;

(iii) for all angular rotation speeds $0<\omega<\omega_{n}$ we have the strict ordering

$$
U_{1}^{+}(\omega)>U_{2}^{+}(\omega)>\ldots>U_{n}^{+}(\omega)>0>U_{n}^{-}(\omega)>\ldots>U_{2}^{-}(\omega)>U_{1}^{-}(\omega)
$$

see Figure 6.

The results of theorem 2 are based on a center manifold analysis after regularization of the degenerate pendulum system (45). Rewriting (45) as a 
system for $U$ and $V:=U_{s}$, and multiplying the right hand side by the Euler multiplier $\kappa=\Gamma(U)$ to eliminate the vanishing denominator $\Gamma(c)=0$, we obtain

$$
\begin{aligned}
\dot{U} & =\Gamma \cdot V \\
\dot{V} & =-\Gamma_{U} \cdot V(\omega-V)-\Gamma^{3} U .
\end{aligned}
$$

Equilibria sit at $U=0$ and at $U=c$, where $\Gamma=0$, each together with $V=0$. Under our assumptions $\Gamma_{U}<0<\omega$ the equilibrium at $(U, V)=(0,0)$ is an unstable node or focus. The equilibrium at $(U, V)=(c, 0)$, in contrast, possesses one zero eigenvalue and one negative eigenvalue. Finite-dimensional center manifold analysis, much in the spirit of section 2.1 above, produces a center manifold $\mathcal{M}$ which is given as a graph

$$
V=\Phi(\omega, U)=\frac{c}{\omega} \Gamma_{U}(c)^{2}(c-U)^{3}+\ldots
$$

near $U=c, V=0$.

It turns out that all solutions of theorem 2 must lie in this center manifold, under just the boundedness and sign conditions imposed there. As an easy consequence all such solutions are asymptotically Archimedean in the far field $s \rightarrow+\infty$. Indeed this follows directly from the first order ODE

$$
U_{s}=V=\Phi(\omega, U)=\frac{c}{\omega} \Gamma_{U}(c)^{2}(c-U)^{3}+\ldots
$$

Substituting $\kappa_{s}=\Gamma_{U}(c) \cdot U_{s}+\ldots$ and $\left(\Gamma_{U}(c) \cdot(c-U)\right)^{3}+\cdots=-\Gamma^{3}=-\kappa^{3}$ for $U$ near $c$ and for small $\kappa$, we obtain

$$
\kappa_{s}=-\frac{c}{\omega} \kappa^{3}+\ldots
$$

in the far field $\kappa \rightarrow 0$. For $s \rightarrow+\infty$ this of course implies

$$
\kappa(s)=a s^{-1 / 2}+\ldots, \quad \text { with } \quad a=\left(\frac{\omega}{2 c}\right)^{1 / 2} .
$$

The asymptotics (53) for the curvature $\kappa$ readily identifies Archimedean spirals. Indeed, arc length parametrization of the position vector $z=Z(t, s)$ in (33) implies that we can write the unit vector $Z_{s}(t, s)$ in the form

$$
Z_{s}(t, s)=\exp (-i w(t, s)) Z_{s}(t, 0)
$$

for a suitable real angle variable $w(t, s)$. Recalling the definition $\kappa i Z_{s}=-Z_{s s}$ of curvature, from (34), we observe that

$$
w(t, s)=\int_{0}^{s} i Z_{s s} / Z_{s} \mathrm{~d} \sigma=\int_{0}^{s} \kappa \mathrm{d} \sigma .
$$

In the present case, where $\kappa$ does not depend on $t$, we obtain the explicit expansion 


$$
w(s)=2 a s^{1 / 2}+\ldots
$$

for $s \rightarrow+\infty$, from (53). Elementary integration of (54), once again, provides the explicit expansion

$$
Z(t, s)=Z_{s}(t, 0) \cdot\left(\frac{i}{a} s^{1 / 2} e^{-2 i a s^{1 / 2}}+\ldots\right) .
$$

Writing $Z=r \exp (i \varphi)$ in polar coordinates $(r, \varphi)$, expansion (57) readily identifies $Z$ to be asymptotically Archimedean with

$$
\lim _{s \rightarrow \infty} \frac{\mathrm{d} r}{\mathrm{~d} \varphi}=-\frac{1}{2 a^{2}}=-\frac{c}{\omega} .
$$

In particular, this also determines the asymptotic wave length in the far field to be $2 \pi c / \omega$, as claimed. The minus sign in (58) indicates that the Archimedean spiral $Z$ is indeed right winding in outward direction.

We now show that the rotation speed $\tilde{\omega}$ of the rigidly rotating spiral $Z(t, s)=\exp (i \tilde{\omega} t) Z(s)$ indeed coincides with the integration constant $\omega$ introduced in (42) above. The normal velocity $U$ is given by the scalar product with the normal vector $\underline{n}$, in complex notation and polar coordinates, as

$$
U=\left(Z_{t}, \underline{n}\right)=\left(i \tilde{\omega} Z, i Z_{s}\right)=\tilde{\omega} \operatorname{Re}\left(Z \cdot \bar{Z}_{s}\right)=\tilde{\omega} r r_{s} .
$$

We now argue in the far field $s \rightarrow \infty$, where $U \rightarrow c$ and $\kappa=\Gamma(U) \rightarrow 0$. In particular, (59) implies

$$
\lim _{s \rightarrow \infty} r r_{s}=c / \tilde{\omega},
$$

and $r_{s} \rightarrow 0$ because $r \rightarrow \infty$. On the other hand, $\left(r \varphi_{s}\right)^{2}+r_{s}^{2}=1$ holds for arc length parametrization, and implies $\lim _{s \rightarrow \infty} r \varphi_{s}= \pm 1$. Therefore the right winding $\varphi_{s}<0$ and the asymptotics (58) imply that

$$
\lim _{s \rightarrow \infty} r r_{s}=\lim _{s \rightarrow \infty} r \frac{\mathrm{d} r}{\mathrm{~d} \varphi} \cdot \varphi_{s}=c / \omega .
$$

Together, (60) and (61) prove $\omega=\tilde{\omega}$ is the rotation frequency, indeed.

We conclude this section with some remarks on the relation of our bifurcation analysis of (45) with the original problem (42). Expressing the left center manifold $\mathcal{M}$ of the equilibrium $(U, V)=(c, 0)$ of (49) by $V=\Phi(\omega, U)$, as in (50), the bifurcation diagram of Figure 6 is the solution set of the equation

$$
\omega=\Phi(\omega, U(0)) .
$$

Indeed $V(0)=U_{s}(0)=\omega-G \kappa_{0}=\omega$ for $G=0$, by (46). The different branches of the bifurcation diagram correspond to the different intersection points of the line $V=\omega$ with the center manifold $\mathcal{M}$ in the phase portrait of Figure $7(\mathrm{a})$. The original boundary condition

$$
\kappa(0)=\kappa_{0}=\Gamma(U(0))
$$


(a)

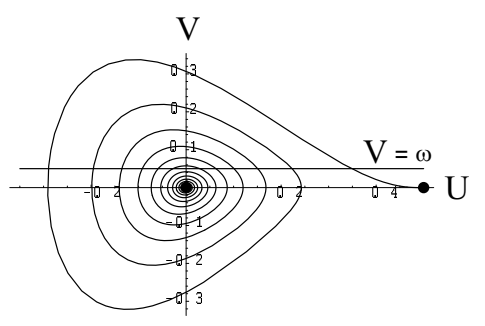

(b)

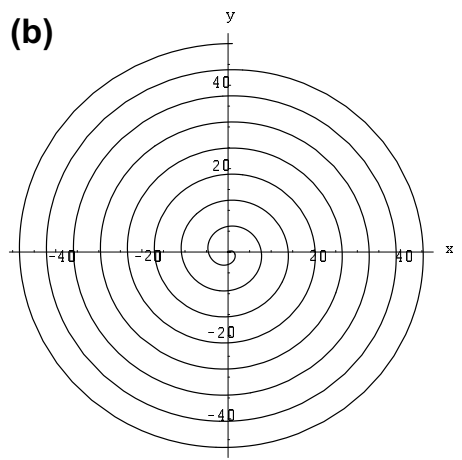

Fig. 7. Archimedean spiral (b) for $\Gamma(U)=1-2 U, \omega=0.05$. For the underlying center manifold in the plane $(U, V)=\left(U, U^{\prime}\right)$ see (a).

can easily be satisfied: we only have to rescale the vertical $U$-axis of Figure 6 by the diffeomorphism $\Gamma$ to represent $\kappa_{0}$. The horizontal $\omega$-axis then provides all rotation frequencies, if any, of rigidly rotating wave solutions with prescribed Dirichlet boundary condition $\kappa=\kappa_{0}$ at the spiral tip. As a caveat, we add that some of the rigidly rotating spirals in the global bifurcation diagram of Figure 6, although asymptotically Archimedean in the far field $r, s \rightarrow \infty$, turn out to be self-intersecting. See [FGT06] for further discussion.

For nonzero $G$, similarly, the relevant bifurcation diagram corresponds to the solution set of

$$
\omega-G \cdot \Gamma(U(0))=\Phi(\omega, U(0)),
$$

again with $U(0)=\Gamma^{-1}\left(\kappa_{0}\right)$ and with $\Phi$ representing the various branches $V=\Phi(\omega, U)$ of the same center manifold $\mathcal{M}$ of (49). We omit a detailed analysis of this case. Instead, we note how $G$ affects the angle $\gamma$ at which the spiral emanates from the circle of its tip motion. An elementary trigonometric calculation at the tip shows

$$
\tan \gamma=-U(0) / G=-\Gamma^{-1}\left(\kappa_{0}\right) / G .
$$

The spiral therefore emanates perpendicularly if, and only if, $G=0$.

\subsection{Eikonal meanders and drifts}

We return to Wiener and Rosenblueth [WR46] in this section to study the propagation of spirals under periodically forced normal velocity

$$
U=U(t, \kappa)=c(t)>0 ;
$$

see (35), (37). We review [MDZ94] and present new results from [Jan06]. Under the modeling assumptions (T1), (T2), (T3) of section 3.1 we obtain meandering and drifting motions of the spiral tip. For simplicity of presentation, we assume the normal velocity $U$ to not depend on curvature. Differently 
from section 3.2, where the resulting equation (40) for our curvature flow was essentially parabolic, we now obtain an eikonal equation familiar from geometric optics. Throughout this section we consider the case of positive tangential tip velocity $G$, where the tangential tip motion extends the curve. Unlike [MDZ94] we do not impose restrictive assumptions like $G(t)=G_{0}(t)-\gamma \kappa_{0}(t)$, $\gamma=$ const $>0$. As we will see, spiral wave solutions then correspond to smooth regular solutions of the resulting nonlinear hyperbolic equation. We will have to consider growing solutions, however, and their response to periodic forcing.

Equation (40) now becomes

$$
\kappa_{t}+c(t)\left(\kappa \int_{0}^{s} \kappa \mathrm{d} \sigma\right)_{s}+G(t) \kappa_{s}=0,
$$

again with Dirichlet boundary condition $\kappa(t, 0)=\kappa_{0}(t)>0$ at $s=0$. As in our discussion (54)-(58) of resulting spiral shapes $Z(t, s)$, we introduce the negative tangent angle $w=\int_{0}^{s} \kappa \mathrm{d} \sigma$, and rewrite (67) as

$$
\begin{aligned}
w_{t}+(c(t) w+G(t)) w_{s} & =G(t) \kappa_{0}(t) \\
w(t, 0) & =0 .
\end{aligned}
$$

Note how the boundary condition $w_{s}(t, 0)=\kappa(t, 0)=\kappa_{0}(t)$ follows from (68), for $G(t) \neq 0$. We are interested in time-periodic forcing functions $c(t), G(t)$, $\kappa_{0}(t)$. The characteristics of the nonlinear hyperbolic balance law (68) satisfy

$$
\begin{aligned}
\dot{s} & =c(t) w+G(t) \\
\dot{w} & =G(t) \kappa_{0}(t)
\end{aligned}
$$

with ${ }^{\cdot}=\mathrm{d} / \mathrm{d} t, t \geq 0, s \geq 0$. In the following we discuss the case of positive $c$, $G, \kappa_{0}$. For other signs see section 4 .

In case $G(t)>0$, for all $t$, the characteristics at the boundary $s=0$, where $w=0$, are pointing inwards towards $s>0$. We can therefore propagate the Dirichlet boundary data (68) to obtain the explicit solution

$$
\begin{aligned}
w\left(t, s\left(t, t_{0}\right)\right) & =\int_{t_{0}}^{t} G(\tau) \kappa_{0}(\tau) \mathrm{d} \tau, \quad \text { at } \\
s\left(t, t_{0}\right) & =\int_{t_{0}}^{t}\left(G(\tau)+c(\tau) \int_{t_{0}}^{\tau} G\left(\tau^{\prime}\right) \kappa_{0}\left(\tau^{\prime}\right) \mathrm{d} \tau^{\prime}\right) \mathrm{d} \tau,
\end{aligned}
$$

parametrized by $0 \leq t_{0} \leq t$. Note that $\left(t, t_{0}\right) \mapsto\left(t, s\left(t, t_{0}\right)\right)$ is a diffeomorphism onto its image, because $c, G, \kappa_{0}>0$. Hence (70) indeed defines $w$ uniquely in the region $\mathcal{T}$ above the characteristic $s_{0}(t):=s(t, 0)$; see Figure 8. Moreover, $T$-periodicity of $G$ and $\kappa_{0}$ implies $s\left(t+T, t_{0}+T\right)=s\left(t, t_{0}\right)$, and therefore $T$-periodicity of the solution $w$ holds in $\mathcal{T}$ :

$$
w(t+T, s)=w(t, s), \quad \text { for } s \leq s_{0}(t) .
$$




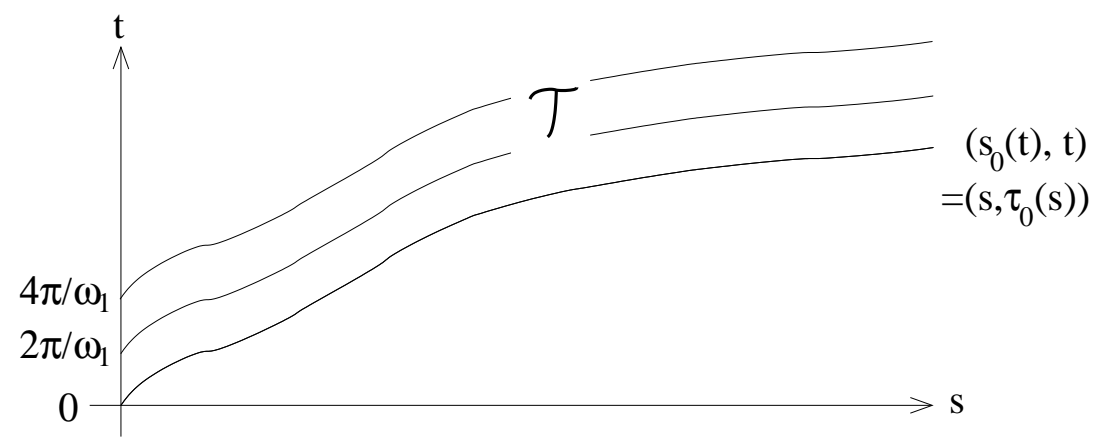

Fig. 8. Characteristics of (68) in $t \geq 0, s \geq 0$ for $G>0$.

Below the characteristic $s_{0}(t)$, only, the initial conditions $w(0, s)=\int_{0}^{s} \kappa(0, \sigma) \mathrm{d} \sigma$ are relevant.

To avoid shock solutions, we assume

$$
w_{s}(0, s)=\kappa(0, s)>0 .
$$

It is easy to see that this condition, which prevents shocks to occur, locally, propagates globally. Indeed we only have to differentiate (68) with respect to $s$. We then observe that $y:=w_{s}$ propagates along the same characteristics (69) as $w$ itself, and satisfies $\dot{y}=-c(t) y^{2}$ with $c>0$.

We conclude that any solution $w(t, s)$ coincides with the unique periodic solution of (68), as soon as $s_{0}(t) \geq s$, independently of the initial condition. This is a very strong stability statement of finite time convergence, uniformly on bounded subsets $s$. For further details see [Jan06].

To render our convergence statement more quantitative and, at the same time, exhibit the Archimedean spiral character of our solutions, we now address the asymptotics of the characteristic $s_{0}(t)$. We claim

$$
\tau_{0}(s)=\left(\frac{2}{\langle c\rangle\left\langle G \kappa_{0}\right\rangle}\right)^{1 / 2} s^{1 / 2}+\ldots
$$

for the inverse function $\tau_{0}:=s_{0}^{-1}$, where $\langle\cdot\rangle$ denotes time average over period $T$.

Indeed, $\int_{0}^{t} a(\tau) \mathrm{d} \tau=\langle a\rangle t+\ldots$ for any periodic function $a$. The remainder is bounded periodic. Applying this simple observation to $s_{0}(t)=s(t, 0)$ in (70), twice, we obtain

$$
s_{0}(t)=\frac{1}{2}\langle c\rangle\left\langle G \kappa_{0}\right\rangle t^{2}+\ldots
$$

with a remainder of order $t$. This proves (73).

To exhibit the Archimedean spiral character of the $T$-periodic solution $w$, we evaluate $w(t, s)$ along characteristics as in (70). Because $w(t+k T, s)=$ 
$w(t, s)$, it suffices to restrict attention to the fundamental band $\tau_{0}(s) \leq t \leq$ $\tau_{0}(s)+T$, where $0 \leq t_{0} \leq T$. The relevant asymptotics of $w$ is therefore given by

$$
\begin{aligned}
w(t, s) & =w\left(\tau_{0}(s), s\right)+\ldots=\left\langle G \kappa_{0}\right\rangle \tau_{0}(s)+\ldots \\
& =\left(2\left\langle G \kappa_{0}\right\rangle /\langle c\rangle\right)^{1 / 2} s^{1 / 2}+\ldots
\end{aligned}
$$

with bounded remainder. Extending the above estimate to include $s$-derivatives we obtain the curvature asymptotics

$$
\kappa(t, s)=w_{s}(t, s)=\left(\frac{1}{2}\left\langle G \kappa_{0}\right\rangle /\langle c\rangle\right)^{1 / 2} s^{-1 / 2}+\ldots
$$

By the curvature analysis of section 3.2, (53)-(61) this shows that our periodic solution $w(t, s)$ represents a periodically fluctuating Archimedean spiral of time independent asymptotic (average) rotation frequency

$$
\omega=\left\langle G \kappa_{0}\right\rangle
$$

and asymptotic wavelength $2 \pi\langle c\rangle /\left\langle G \kappa_{0}\right\rangle$ in the far field.

It may be worth interpreting the characteristic front $s_{0}(t)$ in the Archimedean spiral geometry. Let $r(t)=r\left(t, s_{0}(t)\right)$ denote the distance of the characteristic point on the spiral from its origin. For large $s, t$, we then have a radial propagation speed $\dot{r}$ of the stability zone which is given by

$$
\frac{1}{2} \frac{\mathrm{d}}{\mathrm{d} t} r^{2}=r \dot{r}=r r_{s} \dot{s}_{0}+\ldots=\frac{\langle c\rangle^{2}}{\omega}\left\langle G \kappa_{0}\right\rangle t+\ldots
$$

Here we have used (74) and, for the asymptotically rigidly rotating spiral, also (61). Moreover we have omitted the term $r r_{t}$ which is zero under rigid rotation. With (77), integration of (78) yields

$$
\dot{r}=\langle c\rangle+\ldots
$$

This perhaps intuitive result asserts that the stable core region of the spiral synchronizes the far field at the average radial speed of propagation of the spiral itself. For further details see [Jan06].

What is the resulting tip dynamics, then? In the notation of section 3.2 , let $z(t)=Z(t, 0)$ denote the tip position and $\exp (i \alpha(t)):=Z_{s}(t, 0)$ the tip tangent. Then

$$
\begin{aligned}
\dot{\alpha} & =G(t) \kappa_{0}(t) \\
\dot{z} & =e^{i \alpha}(i c(t)-G(t)) .
\end{aligned}
$$

Indeed, $\dot{z}$ results from the normal and tangential velocities $c$ and $G$ which are rotated by $\alpha$ into the appropriate coordinate frame. Since the normal velocity $c(t)$ is identically constant along the curve, the only contribution to the angle 
change $\mathrm{d} \alpha$ comes from the extension of the curve by an arc of curvature radius $1 / \kappa_{0}$ at the tip and of length $G \mathrm{~d} t$. This provides the equation for $\dot{\alpha}$. It is perhaps helpful to compare this derivation with the rigidly rotating case of (46), where the rotation frequency $\omega$ - alias $\dot{\alpha}-$ was given by the sum of $G \kappa_{0}$ and the now vanishing term $U_{s}(0)$.

Comparing with section 2.4 we see how the tip dynamics (80) takes on the skew product form (12). Indeed we only have to assume the variable $v$ to be $T$-periodic, as Hopf bifurcation had caused it to be there, and our current tip dynamics (80) fits right in. Defining $\omega_{*}:=\left\langle G \kappa_{0}\right\rangle$, the analysis (20), (21) of meanders and drifts applies with

$$
\sigma(t):=(i c(t)-G(t))\left\langle G \kappa_{0}\right\rangle /\left(G(t) \kappa_{0}(t)\right)
$$

of frequency $\omega_{1}=2 \pi / T$.

In summary, we have shown that the periodically forced eikonal flow (67) exhibits Archimedean spirals with very strongly stable meandering and drifting tip motions, if the normal velocity $c(t)$, the tangent tip speed $G(t)$, and the tip curvature $\kappa_{0}(t)$, are all positive [PGP93, MDZ94, Jan06].

As mentioned in the introduction, meandering and resonant drift of spiral waves in the photosensitive BZ reaction can be achieved experimentally by a periodically changing light intensity [BE93b, ZSM94]. The Doppler effect imposes superspiral structures in the case of spiral wave dynamics other than rigid rotation. These superspiral structures have been observed in experiments; see for example [PAV ${ }^{+}$91, LOPS96, BOF97, ZO00, OSL00]. See [SS01] for a mathematical analysis based on linearized analysis and eigenfunctions.

Numerical evidence for superspirals in the kinematic theory has first been presented in [PGP93]. The normal velocity $U$ and the tangential tip velocity $G$ have been assumed to be curvature dependent and a meandering tip trajectory was prescribed, a priori. Superspiral patterns have then been observed, numerically, via a stepwise reconstruction of the spiral wave shape.

In our setting of curvature independent but time dependent normal velocity $U=U(t)=c(t)$, as in (66), the analysis of the eikonal equation (67) reveals superspirals if the normal velocity $c(t)$, the tangent tip speed $G(t)$ or the curvature at the tip $\kappa_{0}(t)$ are positive and time periodic near resonance. To be explicit, we choose for example $c(t)=1+\varepsilon \sin (t), G(t)=\delta c(t)$ and $\kappa_{0}(t)=1 / c(t)$ with $\varepsilon=0.3$ and $\delta=1.1$ or $\delta=1$. Note that $\delta=G \kappa_{0}$ denotes the rotation frequency of the spiral, via $\dot{\alpha}=\delta$ in (80), and the forcing frequency is fixed to be 1 , via the term $\sin (t)$. Then equations $(67)$ and (80) can be solved analytically. For resulting tip motions and superspiral patterns see Figure 9.

Even for periodic functions $c(t), G(t)$ and $\kappa_{0}(t)$ more complicated tip motions and superspiral structures of higher order can be prescribed. Choose for example $c(t)=1+2 \varepsilon \sin (t)+3 \varepsilon \sin (1.1 t), G(t)=c(t) / 5, \kappa_{0}(t)=1 / G(t)$ with $\varepsilon=0.15$. The rotation frequency is thus fixed at 1 , because $\dot{\alpha}=G \kappa_{0}=1$. The near resonant component of $c(t)$ with forcing frequency 1.1 is expected to produce a superspiral, as in the left part of Figure 9. The resonant component 

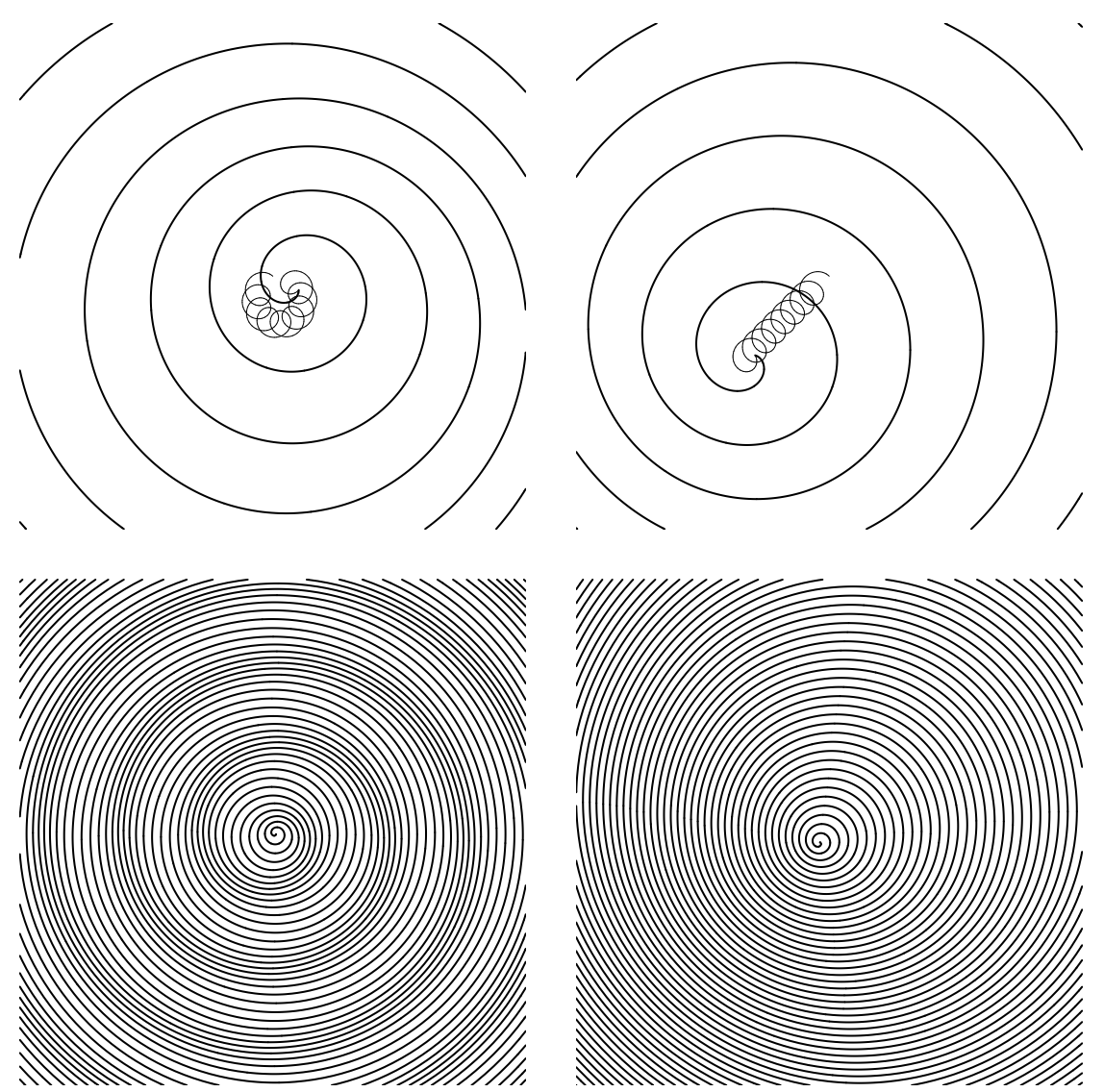

Fig. 9. Meandering superspiral for $\delta=1.1$ (left) and drifting spiral for $\delta=1$ (right) with tip dynamics in the close-up view (top) and Doppler effect (bottom). Forcing is at frequency 1 , near-resonant to the rotation frequency $\delta$.

of $c(t)$ with frequency 1 superimposes a linear drift with an associated Doppler effect on, both, the spiral and the superspiral as in the right part of Figure 9. We choose initial conditions $z(0)=0$ for the tip position and $\alpha(0)=0$ for the tip tangent angle. Solving equation (80) yields exactly: 

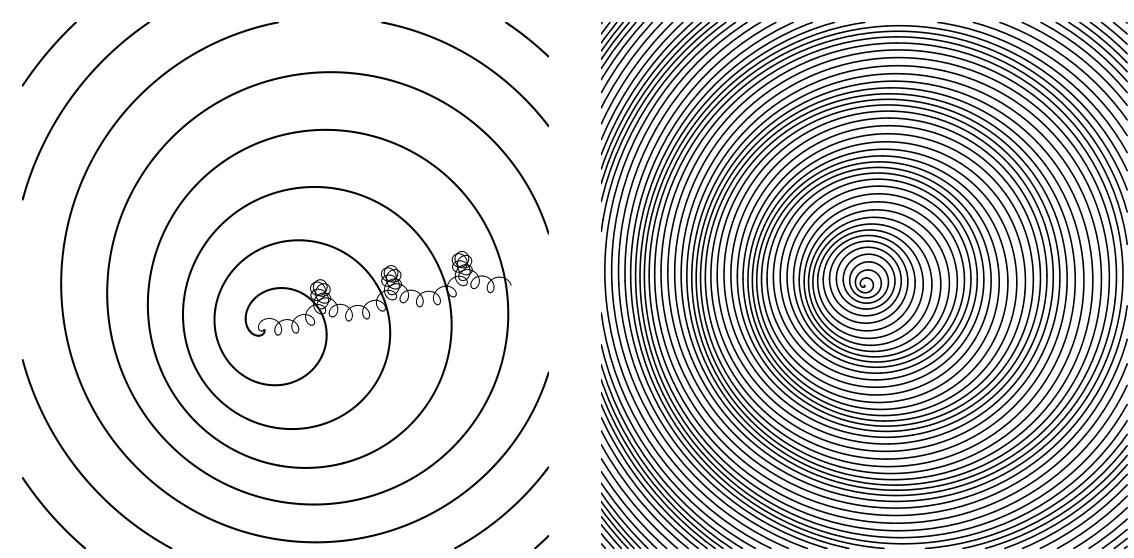

Fig. 10. Drifting superspiral: Meandering and drifting spiral with tip dynamics in the close-up view (left) and Doppler effect (right).

$$
\begin{aligned}
& \alpha(t)=t \\
& z_{1}(t)=-\frac{2081}{1400}-\frac{3}{20} t-\frac{9}{4} \sin (0.1 t)-\frac{1}{5} \sin (t)+\frac{3}{40} \sin (2 t)+\frac{3}{28} \sin (2.1 t) \\
& +\frac{9}{20} \cos (0.1 t)+\cos (t)+\frac{3}{200} \cos (2 t)+\frac{3}{140} \cos (2.1 t) \\
& z_{2}(t)=\frac{125}{56}-\frac{3}{100} t-\frac{9}{20} \sin (0.1 t)+\sin (t)+\frac{3}{200} \sin (2 t)+\frac{3}{140} \sin (2.1 t) \\
& -\frac{9}{4} \cos (0.1 t)+\frac{1}{5} \cos (t)-\frac{3}{40} \cos (2 t)-\frac{3}{28} \cos (2.1 t) \text {. }
\end{aligned}
$$

An explicit expression for the evolution of the curve $Z\left(t, s\left(t, t_{0}\right)\right)$ along the characteristics can be derived from (70); for a visualization see Figure 10. The two different Fourier frequencies 1 and 1.1 of the forcing in $c(t)$, indeed, cause superimposed meandering and drifting tip dynamics, see the tip trajectory in Figure 10. In the far field we recognize the superspiral structure induced by the meandering of the tip, which is drifting due to resonance. Similar observations apply to quasi-periodic superspirals supported by an incommensurate 2 -frequency forcing. Superspirals of infinite order can then be achieved, due to the resulting arbitrarily small difference frequencies. For further analysis see $[\operatorname{Jan} 06]$.

Can the tip dynamics be designed, arbitrarily? Linearly dependent normal and tangential tip velocities, $c(t)=$ const $\cdot G(t)$, and a constant tip curvature $\kappa_{0}$ admit rigid rotation, only. This follows by integration of (80), independently of the choices for $c(t), G(t)$. For $\kappa_{0}(t)=1 / G(t)$, on the other hand, we can prescribe the spiral path, arbitrarily. For example, choose $c(t)=1-0.1 \sin (\pi / 2 h(t)-t), G(t)=0.2-0.1 \cos (\pi / 2 h(t)-t)$. At any time $t$, the control function $h(t)$ assigns a tip drift which superimposes the 

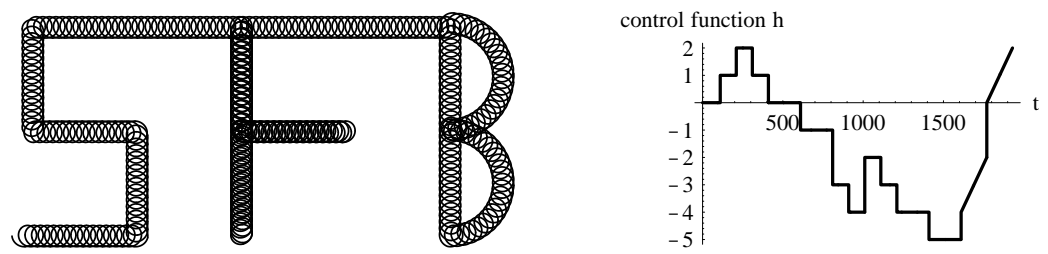

Fig. 11. Control of the spiral wave dynamics: Tip dynamics (left) induced by control function $h(t)$ (right).

rotational dynamics. For a control function $h(t)$ as sketched in Figure 11, we obtain a desired "SFB".

\section{Concluding remarks}

We first comment on several aspects of eikonal meanders; see section 3.3. Specifically we mention the relation to tip control experiments $\left[\mathrm{ZBB}^{+} 04\right.$, ZE04, ZBBE05, KM05, ZE06], discuss the occurrence and interpretation of shock waves, the role of the signs of $G$ and $\kappa_{0}$, possible generalizations to dynamics of scroll wave filaments in three dimensions, and the role of diffusion and viscous regularization in the sense of section 3.2. We then return to the reaction-diffusion view point of section 2 and discuss the chances of a reduced eikonal description of autonomous meanders by relative Hopf bifurcation in the Euclidean group $S E(2)$.

To describe control of tip motion, in the spirit of $\left[\mathrm{ZBB}^{+}\right.$04, ZE04, ZBBE05, KM05, ZE06], we return to the tip dynamics

$$
\begin{aligned}
\dot{\alpha} & =G(t) \kappa_{0}(t) \\
\dot{z} & =e^{i \alpha}(i c(t)-G(t)) .
\end{aligned}
$$

as derived in (80); see also (20), (21). Our derivation did not depend on time periodicity of $c, D, \kappa_{0}$, of course. Control of $z$ can now be effected as follows. By external lighting, the triple $\lambda:=\left(c, G, \kappa_{0}\right) \in\left\{\lambda_{0}, \lambda_{1}\right\}$ is set to two different values $\lambda_{0}$ and $\lambda_{1}$, depending on time. Mostly $\lambda=\lambda_{0}$ with corresponding rigid rotation of $(\alpha, z)$. When the wave front passes a sensor point $z_{0}$, lighting is changed to $\lambda_{1}$, briefly, and then resumes $\lambda_{0}$. As $(\alpha, z)$ pass through their $\lambda_{0}$ cycle, a small offset $\mathrm{d} z$ is thus effected during each period. The offsets add up to a superimposed drift

$$
\frac{\mathrm{d} z}{\mathrm{~d} \tau}=f\left(z-z_{0}\right)
$$

on a slow time scale $\tau$. Here $f(z):=\exp \left(i r+\beta \varphi+a_{0}\right)$ for polar coordinates $z=\exp (i \varphi)$. Indeed, $f$ reflects the asymptotically Archimedean character of the spiral expressed by $G \kappa_{0} r+c \varphi=$ const., with values $\lambda_{0}$; see also (58). 


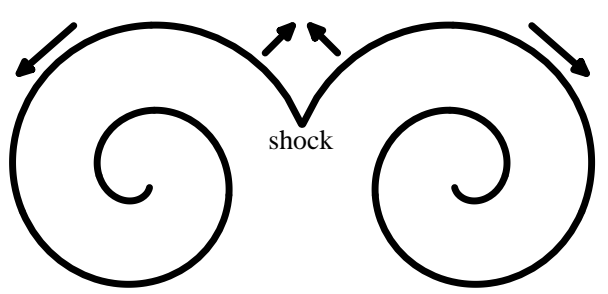

Fig. 12. Collision of spirals and shocks in solutions $w$ of balance laws (68).

The constant $a_{0}$ measures the resulting offset between lighting $\lambda_{0}$ and $\lambda_{1}$. Similarly, triggering lighting at several sensor positions $z_{k}$ superimposes to a slow drift

$$
\frac{\mathrm{d} z}{\mathrm{~d} \tau}=\sum_{k} f\left(z-z_{k}\right)
$$

with correspondingly richer possibilities for control. Alternatively, and more efficiently, active control can be based on the temporal pattern of sensor signals, within one period, to explicitly reconstruct - and control - position $z(t)$ and phase $\alpha(t)$ of the spiral tip.

Balance laws (68) are known to exhibit shock waves, i.e. solutions $w$ with discontinuities of the (negative) tangent angle $w$ at shock positions $s(t)$. See Figure 12 and, for some qualitative remarks, also [Pis06]. The RankineHugoniot condition specifies the shock speed $\dot{s}(t)$ to satisfy

$$
\dot{s}=G+\frac{1}{2} c \frac{\left[w^{2}\right]}{[w]},
$$

where $[w]=w_{+}-w_{-}$measures the jump of $w$ from the left value $w_{-}$to the right value $w_{+}$at the discontinuity. The Lax entropy condition requires $[w]<0$, for positive $c$. See for example [Lax73, Smo94] and the references there. In (72) we have excluded shocks, imposing the initial condition $w_{s}>0$. At collision of spirals, however, shocks may occur; see Figure 12. Indeed (54) then indicates a jump in the tangent direction $Z_{s}(t, s)=\exp (-i w(t, s)) Z_{s}(t, 0)$ of the joined spiral-antispiral curve $Z(t, s)$ at the interface of the two counterrotating spirals. The interface evolves according to the Rankine-Hugoniot condition (86) until the shock strength $[w]$ has decayed to zero and the solution regains regularity.

In section 3 above we have imposed positive sign conditions $c, G, \kappa_{0}>0$. Other combinations of signs are of course conceivable. We only mention the case $c, G<0<\kappa_{0}$ briefly. Reversing time, $t \mapsto-t$, then converts the characteristics to the previous ones of section 3.3; see (69). Therefore asymptotically Archimedean spirals still exist, as a periodic response to the periodic forcing by $c, G, k_{0}$. However, they now rotate backwards compared to the previous case. Moreover, these spirals are now extremely unstable, just as they were extremely stable before. Indeed, any perturbation in the far field now propagates 
inwards at negative radial velocity $\dot{r}=\langle c\rangle+\ldots$, see (79), and destructively reaches the spiral tip after some finite time. Afterwards the Archimedean spiral disappears and the initial conditions reign. This may account for far-field break-up of spiral waves.

The eikonal approach of section 3.3 suggests generalizations to scroll wave dynamics in three space dimensions. For beautiful experimental results based on computer tomography see [BS06]. For numerical simulations in an excitable media reaction-diffusion setting see [FM00], for example, and the references there. For experimental and numerical investigations of chemical turbulence of scroll waves see [AKMS04, ASM06, MS06]. Indeed we may consider an embedded oriented surface $S$ in $\mathbb{R}^{3}$, called a scroll wave, with one-dimensional boundary curve $\gamma$, called a filament. Of course, the surface $S$ generalizes our rotating planar curve $Z(t, s)$, and the curve $\gamma$ corresponds to the tip $z$ of $Z$. We may then propagate $S$ and its boundary $\gamma$ with speed $c$ in the (oriented) normal direction. The propagation of the boundary curve $\gamma$ will also occur with speed $G$ along the outward tangent of $S$, and with prescribed sectional curvature of $S$. Several variants of mean or Gauss curvature flows may also be invoked to generalize the curvature dependence of normal velocity to the surface $S$, see also [Mik95, ASM06]. As a result, meandering, drifting, and even colliding scroll wave filaments should be observed.

Eikonal curvature flow precludes relative Hopf bifurcation. Indeed the resulting tip dynamics

$$
\begin{aligned}
\dot{\alpha} & =G \kappa_{0} \\
\dot{z} & =e^{i \alpha}(i c-G) .
\end{aligned}
$$

of (80) generates purely exponential dynamics of $(\exp (i \alpha), z) \in S E(2)$ with circular motion, for $G \kappa_{0} \neq 0$. This excludes meanders and drifts. At present, it is unknown whether the curvature dependent normal velocities $U=U(\kappa)$ of section 3.2 are able to remedy this modeling difficulty. It only seems clear that affine linear dependencies $U(\kappa)=c-D \kappa$ as in (38) will not remedy the problem, as long as $D>0$ remains small. Indeed small $D$ act as a viscous regularization in (40) and, analogously, for the balance law (68). For monotone solutions $w_{s}=\kappa>0$ which are regular, however, small positive $D$ act as a regular perturbation which should not be strong enough to produce meanders and drifts which are absent for $D=0$.

An easy remedy for this problem would be the introduction of additional "hidden" variables which influence the dynamics of the curve, notably via $c$, $D, G$, or $\kappa_{0}$. A modification of $G$ or $\kappa_{0}$ at the tip alone, for example, might be sufficient. Specifically we may introduce a hidden scalar variable $v$ at the tip, alone, together with some hypothetical interaction dynamics

$$
\begin{aligned}
\dot{\kappa}_{0} & =g\left(\kappa_{0}, v\right) \\
\dot{v} & =h\left(\kappa_{0}, v\right)
\end{aligned}
$$


which undergoes Hopf bifurcation as a (suppressed) parameter is varied. Here $\kappa_{0}=\kappa_{0}(t)$ is supposed to provide the Dirichlet boundary condition (41) for the curvature PDE (40). The resulting periodic fluctuation $\kappa_{0}(t)$ would then drive and synchronize the Archimedean spiral, globally, into meandering and drifting motions.

For the moment, a coupling like (88) remains speculation. Only reactiondiffusion systems as studied in section 2 provide a modeling description which is based on reasonably "first" principles. The mathematically proper approach to autonomous meanders and relative Hopf bifurcation, which are present in the reaction-diffusion setting, would then be a derivation of reduced systems like (88), (40), (41) from reaction-diffusion systems which model excitable media. The relevant techniques, vaguely, are to include singular perturbation theory. But details still require much future effort.

\section{References}

[AKMS04] S. Alonso, R. Kähler, A. S. Mikhailov, and F. Sagués. Expanding scroll rings and negative tension turbulence in a model of excitable media. Phy. Rev. E, 70(5):1-10, 2004.

[Ang90] S. Angenent. Parabolic equations for curves on surfaces. I: Curves with p-integrable curvature. Ann. Math., 132:451-483, 1990.

[Ang91] S. Angenent. Parabolic equations for curves on surfaces. II: Intersections, blow-up and generalized solutions. Ann. Math., 133:171-251, 1991.

[ASM06] S. Alonso, F. Sagués, and A. S. Mikhailov. Periodic forcing of scroll rings and control of Winfree turbulence in excitable media. Chaos, 16:1-11, 2006.

[Bar94a] D. Barkley. Euclidean symmetry and the dynamics of rotating spiral waves. Phys. Rev. Lett., 72:164-167, 1994.

[Bar94b] D. Barkley. Linear stability analysis of rotating spiral waves in excitable media. Phys. Rev. Lett., 68:2090-2093, 1994.

[BBSE00] H. Brandtstädter, M. Braune, I. Schebesch, and H. Engel. Experimental study of the dynamics of spiral pairs in light-sensitive BelousovZhabotinskii media using an open-gel reactor. Chem. Phys. Lett., 322:145-154, 2000.

[BE93a] M. Braune and H. Engel. Compound rotation of spiral waves in a lightsensitive Belousov-Zhabotinsky medium. Chem. Phys. Lett., 204:257264, 1993.

[BE93b] M. Braune and H. Engel. Compound rotation of spiral waves in active media with periodically modulated excitability. Chem. Phys. Lett., 211:534-540, 1993.

[BM03] M. Bertram and A. Mikhailov. Pattern formation on the edge of chaos: mathematical modeling of CO oxidation on a $\mathrm{Pt}(110)$ surface under global delayed feedback. Phys. Rev. E, 67:1-9, 2003.

[BOF97] A. L. Belmonte, Q. Ouyang, and J.-M. Flesselles. Experimental Survey of Spiral Dynamics in the Belousov-Zhabotinsky Reaction. Journal de Physique II, 7:1425-1468, 1997. 
[BS06] T. Bánsági and O. Steinbock. Nucleation and Collapse of Scroll Rings in Excitable Media. Preprint, 2006.

[BT96] P. K. Brazhnik and J. J. Tyson. Nonspiral excitation waves beyond the eikonal approximation. Phys. Rev. E, 54:4338-4346, 1996.

[CM01] F. Cao and L. Moisan. Geometric computation of curvature driven plane curve evolutions. SIAM J. Numer. Anal., 39(2):624-646, 2001.

[FGT04] B. Fiedler, J.-S. Guo, and J.-C. Tsai. Multiplicity of rotating spirals under curvature flows with normal tip motion. J. Differ. Equations, 205(1):211-228, 2004.

[FGT06] B. Fiedler, J.-S. Guo, and J.-C. Tsai. Rotating spirals of curvature flows: a center manifold approach. To appear in Ann. Mat. Pura Appl., 2006.

[FL98] M. Falcke and H. Levine. Pattern Selection by Gene Expression in Dictyostelium Discoideum. Phys. Rev. Lett., 80:3875-3878, 1998.

[FM00] B. Fiedler and R. M. Mantel. Crossover collision of scroll wave filaments. Doc. Math., J. DMV, 5:695-731, 2000.

[FM02] M. Fila and H. Matano. Blow-up in nonlinear heat equations from the dynamical systems point of view. In B. Fiedler (ed.), Handbook of dynamical systems. Volume 2. Amsterdam: Elsevier, 723-758, 2002.

[FS96] B. Fiedler and J. Scheurle. Discretization of homoclinic orbits, rapid forcing and "invisible" chaos. Mem. Am. Math. Soc., 570:79 p., 1996.

[FS03] B. Fiedler and A. Scheel. Spatio-temporal dynamics of reaction-diffusion patterns. In M. Kirkilionis (ed.) et al., Trends in nonlinear analysis. On the occasion of the 60th birthday of Willi Jäger. Berlin: Springer, 23-152, 411-417, 2003.

[FSSW96] B. Fiedler, B. Sandstede, A. Scheel, and C. Wulff. Bifurcation from relative equilibria of noncompact group actions: Skew products, meanders, and drifts. Doc. Math., J. DMV, 1:479-505, 1996.

[FT98] B. Fiedler and D. Turaev. Normal forms, resonances, and meandering tip motions near relative equilibria of Euclidean group actions. Arch. Ration. Mech. Anal., 145(2):129-159, 1998.

[Gel02] V. Gelfreich. Numerics and exponential smallness. In B. Fiedler (ed.), Handbook of dynamical systems. Volume 2. Amsterdam: Elsevier, 265-312, 2002.

[Geo03] M. Georgi. Spiral waves in reaction-diffusion systems - the reduced vector field (Spiralwellen in Reaktions-Diffusions-Systemen - Das reduzierte Vektorfeld). Diploma Thesis, Free University of Berlin, 2003.

[GH86] M. Gage and R. Hamilton. The heat equation shrinking convex plane curves. J. Differ. Geom., 23:69-96, 1986.

[GJ05] M. Georgi and N. Jangle. Spiral wave motion in reaction-diffusion systems. In F. Dumortier, H. Broer, J. Mawhin, A. Vanderbauwhede, and S. V. Lunel, editors, Proceedings Equadiff 03, International Conference on Differential Equations, Hasselt 2003, pages 651-656, Singapore, 2005. World Scientific.

[GL01] V. Gelfreich and V. Lazutkin. Splitting of separatrices: Perturbation theory and exponential smallness. Russ. Math. Surv., 56(3):499-558, 2001.

[Hen81] D. Henry. Geometric theory of semilinear parabolic equations. Lecture Notes in Mathematics. 840. Berlin-Heidelberg-New York: SpringerVerlag, 348 p., 1981. 
[HPS77] M. Hirsch, C. Pugh, and M. Shub. Invariant manifolds. Lecture Notes in Mathematics. 583. Berlin-Heidelberg-New York: Springer-Verlag. 149 p., 1977.

[Hui93] G. Huisken. Local and global behaviour of hypersurfaces moving by mean curvature. In R. Greene (ed.) et al., Differential geometry. Part 1: Partial differential equations on manifolds. Proceedings of a summer research institute, held at the University of California, Los Angeles, CA, USA, July 8-28, 1990. Providence, RI: American Mathematical Society. Proc. Symp. Pure Math. 54, Part 1, 175-191, 1993.

[IIY98] R. Ikota, N. Ishimura, and T. Yamaguchi. On the structure of steady solutions for the kinematic model of spiral waves in excitable media. Japan J. Ind. Appl. Math., 15(2):317-330, 1998.

[Jan03] N. Jangle. Center manifolds in reaction-diffusion systems on unbounded domains (Zentrumsmannigfaltigkeiten in Reaktions-DiffusionsSystemen auf unbeschränkten Gebieten). Diploma Thesis, Free University of Berlin, 2003.

[Jan06] N. Jangle. Dynamics of spatio-temporal patterns in heterogeneous media (Dynamik räumlich-zeitlicher Muster in heterogenen Medien). Dissertation thesis in preparation, 2006.

[JSW89] W. Jahnke, W. Skaggs, and A. Winfree. Chemical vortex dynamics in the Belousov-Zhabotinskii reaction and in the two-variable Oregonator model. J. Chem. Phys., 93:740-749, 1989.

[Kee92] J. P. Keener. The core of the spiral. SIAM J. Appl. Math., 52(5):13701390, 1992.

[KGZM01] O. Kheowan, V. Gaspar, V. Zykov, and S. Müller. Measurements of kinematical parameters of spiral waves in media of low excitability. Phys. Chem. Lett., 3:4747-4764, 2001.

[KM05] O.-U. Kheowan and S. C. Müller. Control of spiral waves in excitable media. Appl. Math. Comput., 164(2):373-390, 2005.

[KT92] J. P. Keener and J. J. Tyson. The dynamics of scroll waves in excitable media. SIAM Rev., 34(1):1-39, 1992.

[Lax73] P. D. Lax. Hyperbolic systems of conservation laws and the mathematical theory of shock waves. CBMS-NSF Regional Conference Series in Applied Mathematics. 11. Philadelphia, Pa.: SIAM, Society for Industrial and Applied Mathematics, 48 p. , 1973.

[LOPS96] G. Li, Q. Ouyang, V. Petrov, and H. L. Swinney. Transition from Simple Rotating Chemical Spirals to Meandering and Traveling Spirals. Phys. Rev. Lett., 77:2105-2108, 1996.

[MB96] R. Mantel and D. Barkley. Periodic forcing of spiral waves in excitable media. Phys. Rev. E, 54:4791-4802, 1996.

[MDZ94] A. Mikhailov, V. Davydov, and V. Zykov. Complex dynamics of spiral waves and motion of curves. Physica D, 70(1-2):1-39, 1994.

[Men91] P. Mendes. A metric property of Cherry vector fields on the torus. $J$. Differ. Equations, 89(2):305-316, 1991.

[Mer92] E. Meron. Pattern formation in excitable media. Physics Reports, 218:1-66, 1992.

[Mik95] A. S. Mikhailov. Three-dimensional Kinematics. Chaos, Solitons and Fractals, 5:673-679, 1995. 
[Mik01] K. Mikula. Solution and application of anisotropic curvature driven evolution of curves (and surfaces). In M. Falcone (ed.) et al., Numerical methods for viscosity solutions and applications. Singapore: World Scientific. Ser. Adv. Math. Appl. Sci. 59, 173-196, 2001.

[Mik03] A. S. Mikhailov. Modeling pattern formation in excitable media: The legacy of Norbert Wiener. In J. Milton, P. Jung (eds.), Epilepsy as a dynamic disease. Biological and Medical Physics Series. Springer, Berlin Heidelberg, 125-163, 2003.

[MR92] P. Moreira and A. Ruas. Metric properties of Cherry flows. J. Differ. Equations, 97(1):16-26, 1992.

[MS95] R. Malladi and J. Sethian. Image processing via level set curvature flow. Proc. Natl. Acad. Sci. USA, 92(15):7046-7050, 1995.

[MS06] A. S. Mikhailov and K. Showalter. Control of waves, patterns and turbulence in chemical systems. Phys. Rep., 425:79-194, 2006.

[MZ91] A. S. Mikhailov and V. S. Zykov. Kinematical theory of spiral waves in excitable media: Comparison with numerical simulations. Physica D, 52:379-397, 1991.

[MZ94] S. C. Müller and V. S. Zykov. Simple and complex spiral wave dynamics. Philos. Trans. R. Soc. Lond., Ser. A, 347:677-685, 1994.

[NvORE93] S. Nettesheim, A. von Oertzen, H. Rotermund, and G. Ertl. Reaction diffusion patterns in the catalytic CO-oxidation on $\mathrm{Pt}(110)$-front propagation and spiral waves. J. Chem. Phys., 98:9977-9985, 1993.

[OSL00] Q. Ouyang, H. L. Swinney, and G. Li. Transition from spirals to defectmediated turbulence driven by a doppler instability. Phys. Rev. Lett., 84:1047-1050, 2000.

$\left[\mathrm{PAV}^{+}\right.$91] V. Pérez-Muñuzuri, R. Aliev, B. Vasiev, V. Pérez-Vilar, and V. Krinsky. Super-spiral structures in an excitable medium. Nature, 353:740-742, 1991.

[PdM82] J. j. Palis and W. de Melo. Geometric theory of dynamical systems. An introduction. Transl. from the Portugese by A. K. Manning. New York - Heidelberg - Berlin: Springer-Verlag, 198 p., 1982.

[Per06a] G. Perelman. Finite extinction time for the solutions to the Ricci flow on certain three-manifolds. Preprint, 2006.

[Per06b] G. Perelman. Ricci flow with surgery on three-manifolds. Preprint, 2006.

[Per06c] G. Perelman. The entropy formula for the Ricci flow and its geometric applications. Preprint, 2006.

[PGP93] V. Pérez-Muñuzuri, M. Gómez-Gesteira, and V. Pérez-Villar. A geometrical-kinematical approach to spiral wave formation: super-spiral waves. Physica D Nonlinear Phenomena, 64:420-430, 1993.

[Pis06] L. Pismen. Patterns and Interfaces in Dissipative Dynamics. Berlin: Springer-Verlag. 369 p., 2006.

[PM95] T. Plesser and K. Müller. Fourier analysis of the complex motion of spiral tips in excitable media. Int. J. Bifurcation Chaos, 5:1071-1084, 1995.

[PZMK00] A. Panfilov, V. Zykov, S. Müller, and J. Keener. Defibrillation of cardiac tissue by multiple subthreshold shocks. Phys. Rev. E, 61:4644-4667, 2000 . 
[RGS ${ }^{+}$96] M. Ruiz-Villarreal, M. Gómez-Gesteira, C. Souto, A. P. Muñuzuri, and V. Pérez-Villar. Long-term vortex interaction in active media. Phys. Rev. E, 54:2999-3002, 1996.

[Sch98] A. Scheel. Bifurcation to spiral waves in reaction-diffusion systems. SIAM J. Math. Anal., 29(6):1399-1418, 1998.

[Shu87] M. Shub. Global stability of dynamical systems. With the collab. of Albert Fathi and Remi Langevin. Transl. from the French by Joseph Christy. New York etc.: Springer-Verlag, 150 p., 1987.

[Smo94] J. Smoller. Shock waves and reaction-diffusion equations. 2nd ed. Grundlehren der Mathematischen Wissenschaften. 258. New York: Springer- Verlag, 632 p., 1994.

[SS91] G. Skinner and H. Swinney. Periodic to quasiperiodic transition of chemical spiral rotation. Physica D, 48:1-16, 1991.

[SS01] B. Sandstede and A. Scheel. Superspiral Structures of Meandering and Drifting Spiral Waves. Phys. Rev. Lett., 86:171-174, 2001.

[SS04] B. Sandstede and A. Scheel. Defects in oscillatory media: towards a classification. SIAM J. Appl. Dyn. Syst., 3(1):1-68, 2004.

[SSW97] B. Sandstede, A. Scheel, and C. Wulff. Dynamics of spiral waves on unbounded domains using center-manifold reductions. J. Differ. Equations, 141(1):122-149, 1997.

[SSW99] B. Sandstede, A. Scheel, and C. Wulff. Bifurcations and dynamics of spiral waves. J. Nonlinear Sci., 9(4):439-478, 1999.

[SV85] J. Sanders and F. Verhulst. Averaging methods in nonlinear dynamical systems. Applied Mathematical Sciences, 59. New York etc.: SpringerVerlag, 247 p., 1985.

[SZM93] O. Steinbock, V. Zykov, and S. C. Müller. Control of Spiral-Wave Dynamics in Active Media by Periodic Modulation of Excitability. Nature, 366:322-324, 1993.

[TK88] J. J. Tyson and J. P. Keener. Singular perturbation theory of traveling waves in excitable media (A review). Physica D, 32(3):327-361, 1988.

[Van89] A. Vanderbauwhede. Centre manifolds, normal forms and elementary bifurcations. Dynamics Reported 2:89-169, 1989.

[VI92] A. Vanderbauwhede and G. Iooss. Center manifold theory in infinite dimensions. In Dynamics Reported, New Series 1:125-163, 1992.

[Win72] A. Winfree. Spiral waves of chemical activity. Science, 175:634-636, 1972.

[Win01] A. T. Winfree. The geometry of biological time. 2nd ed. Interdisciplinary Applied Mathematics. Berlin: Springer, 777 p., 2001.

[WR46] N. Wiener and A. Rosenblueth. The mathematical formulation of the problem of conduction of impulses in a network of connected excitable elements, specifically in cardiac muscle. Arch. Inst. Cardiol. Mexico, 16:205-265, 1946.

[Wul96] C. Wulff. Theory of Meandering and Drifting Spiral Waves in ReactionDiffusion Systems. PhD thesis, FU Berlin, 1996.

$\left[Z_{B B}^{+}\right.$03] V. Zykov, G. Bordiougov, H. Brandtstädter, I. Gerdes, and H. Engel. Periodic forcing and feedback control of nonlinear lumped oscillators and meandering spiral waves. Phys. Rev. E, 68:1-11, 2003.

$\left[Z_{B B}^{+} 04\right]$ V. Zykov, G. Bordiougov, H. Brandtstädter, I. Gerdes, and H. Engel. Global control of spiral wave dynamics in an excitable domain of circular and elliptical shape. Phys. Rev. Lett., 92:1-4, 2004. 
[ZBBE05] V. Zykov, H. Brandtstädter, G. Bordiougov, and H. Engel. Interference patterns in spiral wave drift induced by a two-point feedback. Phys. Rev. E, 72:1-4, 2005.

[ZE00] V. Zykov and H. Engel. Experimental study of the dynamics of spiral pairs in light-sensitive BelousovZhabotinskii media using an open-gel reactor. Chem. Phys. Lett., 323:145-154, 2000.

[ZE04] V. Zykov and H. Engel. Dynamics of spiral waves under global feedback in excitable domains of different shapes. Phys. Rev. E, 70:1-9, 2004.

[ZE06] V. Zykov and H. Engel. Unified approach to feedback-mediated control of spiral waves in excitable media. This volume, 2006.

[ZO00] L. Q. Zhou and Q. Ouyang. Experimental studies on long-wavelength instability and spiral breakup in a reaction-diffusion system. Phys. Rev. Lett., 85:1650-1653, 2000.

[ZSM94] V. Zykov, O. Steinbock, and S. Müller. External forcing of spiral waves. Chaos, 4:509-518, 1994.

[Zyk87] V. Zykov. Simulation of wave processes in excitable media. Transl. from the Russian by Potrans, edited by A. T. Winfree. Nonlinear Science: Theory and Applications. Manchester etc.: Manchester University Press, 235 p., 1987. 